\title{
CDK7 regulates organ size and tumor growth by safeguarding the Hippo pathway effector Yki/Yap/Taz in the nucleus
}

\author{
Yong Suk Cho, ${ }^{1,6}$ Shuang Li, ${ }^{1,6}$ Xiaohui Wang, ${ }^{2,3}$ Jian Zhu, ${ }^{1}$ Shu Zhuo, ${ }^{1}$ Yuhong Han, ${ }^{1}$ Tao Yue, \\ Yingzi Yang, ${ }^{2,3}$ and Jin Jiang, ${ }^{1,5}$ \\ ${ }^{1}$ Department of Molecular Biology, University of Texas Southwestern Medical Center, Dallas, Texas 75390 , USA; ${ }^{2}$ Department of \\ Developmental Biology, Harvard School of Dental Medicine, Boston, Massachusetts 02215, USA; ${ }^{3}$ Harvard Stem Cell Institute, \\ Boston, Massachusetts 02215, USA ${ }_{i}^{4}$ Center for the Genetics and Host Defense, ${ }^{5}$ Department of Pharmacology, University of Texas \\ Southwestern Medical Center, Dallas, Texas 75390, USA
}

\begin{abstract}
Hippo signaling controls organ size and tumor progression through a conserved pathway leading to nuclear translocation of the transcriptional effector Yki/Yap/Taz. Most of our understanding of Hippo signaling pertains to its cytoplasmic regulation, but how the pathway is controlled in the nucleus remains poorly understood. Here we uncover an evolutionarily conserved mechanism by which CDK7 promotes Yki/Yap/Taz stabilization in the nucleus to sustain Hippo pathway outputs. We found that a modular E3 ubiquitin ligase complex CRL4 ${ }^{\text {DCAF12 }}$ binds and targets Yki/Yap/Taz for ubiquitination and degradation, whereas CDK7 phosphorylates Yki/Yap/Taz at S169/ S128/S90 to inhibit CRL4 ${ }^{\text {DCAF12 }}$ recruitment, leading to Yki/Yap/Taz stabilization. As a consequence, inactivation of CDK7 reduced organ size and inhibited tumor growth, which could be reversed by restoring Yki/Yap activity. Our study identifies an unanticipated layer of Hippo pathway regulation, defines a novel mechanism by which CDK7 regulates tissue growth, and implies CDK7 as a drug target for Yap/Taz-driven cancer.
\end{abstract}

[Keywords: CDK7; CRL4; Cul4; DCAF12; Hippo; Taz; Yap; Yki; cancer; organ size]

Supplemental material is available for this article.

Received September 25, 2019; revised version accepted November 25, 2019.

The regulation of tissue growth and organ size during development and regeneration depends on a delicate balance between cell proliferation and cell death, which is precisely controlled not only by environmental cues such as hormonal signals, cytokines, and nutrients, but also by organ-intrinsic mechanisms. The Hippo tumor suppressor pathway, which was initially discovered in Drosophila, has emerged as an evolutionarily conserved signaling pathway that regulates tissue growth and organ size in a wide range of species including humans (Pan 2007; Zhang et al. 2009; Halder and Johnson 2011). Not surprisingly, deregulation of Hippo pathway activity has been implicated in many types of human cancer and other diseases $(\mathrm{Yu}$ et al. 2015; Zanconato et al. 2016b; Zheng and Pan 2019).

The core Hippo signaling pathway contains a kinase cassette with an upstream kinase Hippo (Hpo)/MST1/2 phosphorylating and activating a downstream kinase Warts (Wts)/Lats1/2 (Harvey et al. 2003; Jia et al. 2003; Pantalacci et al. 2003; Udan et al. 2003; Wu et al. 2003). Activated Wts/Lats1/2 in turn phosphorylates the Hippo

${ }^{6}$ These authors contribute equally to this work.

Corresponding author: jin.jiang@utsouthwestern.edu

Article published online ahead of print. Article and publication date are online at http://www.genesdev.org/cgi/doi/10.1101/gad.333146.119. pathway effector Yorkie (Yki) in Drosophila and Yap/Taz in mammals, resulting in cytoplasmic retention of Yki/ Yap/Taz via its interaction with 14-3-3 (Huang et al. 2005; Dong et al. 2007; Zhao et al. 2007; Oh and Irvine 2008; Zhang et al. 2008; Ren et al. 2010). Various upstream signals act through Wts/Lats1/2-dependent and -independent mechanisms to promote translocation of Yki/Yap/ Taz into the nucleus, where it binds to the Hippo pathway transcription factors Scalloped (Sd)/TEAD to regulate genes involved in the control of cell growth, proliferation, survival, and metabolism (Wu et al. 2008; Zhang et al. 2008; Zhao et al. 2008; Koo and Guan 2018; Totaro et al. 2018; Moya and Halder 2019).

How Yki/Yap/Taz is regulated in the nucleus is still poorly understood, but recent studies revealed that phosphorylation by a nuclear kinase PRP4K restricts Yki/Yap/ Taz nuclear localization whereas monomethylation of Yap by Set1A blocks its nuclear export (Cho et al. 2018; Fang et al. 2018). In addition, a recent study demonstrated

(C) 2020 Cho et al. This article is distributed exclusively by Cold Spring Harbor Laboratory Press for the first six months after the full-issue publication date (see http://genesdev.cshlp.org/site/misc/terms.xhtml). After six months, it is available under a Creative Commons License (Attribution-NonCommercial 4.0 International), as described at http://creativecommons.org/licenses/by-nc/4.0/. 
that mechanical signals can promote Yap/Taz activation in the nucleus by dissociating it from a SWI/SNF inhibitory complex (Chang et al. 2018). Here we identified CDK7 as a novel Hippo pathway component that phosphorylates and stabilizes Yki/Yap/Taz in the nucleus. We found that inhibition of CDK7 allows a modular E3 ubiquitin ligase CRL4 ${ }^{\text {DCAF12 }}$ to ubiquitinate nuclear Yki/Yap/Taz, and targets it for proteasome-mediated degradation, leading to down-regulation of Hippo pathway target gene expression, reduced organ size, and diminished tumor growth. Hence, CDK7 functions to safeguard nuclear Yki/Yap/ Taz and serves as a promising drug target for Yap/Tazdriven cancer.

\section{Result}

Inactivation of $C D K 7 / C y c H / M a t 1$ suppresses Yki-driven tissue overgrowth

To identify additional Hippo pathway regulators, we conducted an in vivo RNAi screen to identify enhancers and suppressors of the tissue overgrowth phenotype caused by Yki overexpression in the Drosophila eye (GMR > Yki: GMR-Gal4/UAS-Yki) (Fig. 1A,B; Yue et al. 2012; Cho et al. 2018). We initially screened transgenic RNAi lines targeting the Drosophila kinome and identified CDK7 as a suppressor of the eye overgrowth phenotype caused by GMR > Yki. Knockdown of CDK 7 by three independent transgenic RNAi (UAS-CDK7-RNAi) lines: $C D K 7^{V 10442}, C D K 7^{V 103413}$, and $C D K 7^{B L 62304}$, suppressed the GMR-Yki phenotype in a similar manner (Fig. 1B,C, M; Supplemental Fig. S1G,H). CDK7 $7^{V 10442}$-mediated suppression of the GMR-Yki phenotype was negated by coexpression of a wild-type CDK7 (CDK $\left.7^{\mathrm{WT}}\right)$ but exacerbated by a kinase-dead CDK7, CDK $7^{\mathrm{DR}}$ (D137R) (Supplemental Fig. S1B-E). Furthermore, expression of CDK $7{ }^{\mathrm{WT}}$ promoted, whereas $\mathrm{CDK} 7^{\mathrm{DR}}$ inhibited, Yki-driven eye overgrowth (Fig. 1D,M; Supplemental Fig. S1F), indicating that the kinase activity is essential for CDK7 to promote Yki-driven tissue growth and that $\mathrm{CDK} 7{ }^{\mathrm{DR}}$ acts dominant negatively to interfere with Yki activity. CDK7 RNAi did not suppress eye overgrowth caused by overexpression of a constitutively active form of insulin receptor $(G M R>$ $\left.\operatorname{In} R^{C A}\right)$ or a constitutively active form of $\mathrm{Sd}(G M R>S d$ $G A)$ (Fig. 1I-L), suggesting that CDK7 specifically modulates tissue growth driven by Yki. Consistent with this, CDK7 RNAi blocked GMR > Yki induced expression of a Hippo pathway target gene diap1-lacZ, while coexpression of $\mathrm{CDK} 7{ }^{\mathrm{WT}}$ enhanced it (Fig. $\left.1 \mathrm{~A}^{\prime}-\mathrm{D}^{\prime}\right)$.

CDK7 is a transcriptional kinase and a subunit of the TFIIH complex that phosphorylates polymerase II (Pol-II) C-terminal tail (CTD) to regulate transcription (Fisher 2005). In addition, CDK7 acts as a CDK activating kinase (CAK) to phosphorylate and activate other CDKs required for cell division (Fisher 2005). However, the observation that CDK7 RNAi selectively suppressed eye overgrowth driven by $G M R>Y k i$, but not by $G M R>\operatorname{In} R^{C A}$ or $G M R>$ $S d-G A$, suggests that the growth suppression by CDK7 RNAi was unlikely due to a down-regulation of general transcription or cell cycle progression. Indeed,
$G M R>Y k i$-driven tissue overgrowth was also suppressed in a hypomorphic CDK7 mutant background $\left(C D K 7^{S 164 A / T 170 A}\right)$ in which general transcription and cell cycle progression were unaffected (Fig. 1G,H,N; Larochelle et al. 2001; Morishita et al. 2013). Therefore, under the experimental conditions we used (RNAi and hypomorphic mutation), there was enough residual CDK7 kinase activity to support general transcription and cell cycle progression.

CDK7 forms a complex with cyclin $\mathrm{H}(\mathrm{CycH})$ and Mat 1 that regulate its kinase activity and substrate recognition, respectively (Fisher 2005). Knockdown of either $\mathrm{CycH}$ $\left(\mathrm{CyCH}^{\text {V104312 }}\right.$; $\mathrm{CycH}^{\text {BL34732) }}$ or Mat1 (Mat1 $^{\text {V104780; }}$; Mat1 ${ }^{\text {BL57312 }}$ | also suppressed Yki-driven eye overgrowth similar to CDK7 RNAi (Fig. 1E,F,M; Supplemental Fig. $\mathrm{S} 1 \mathrm{I}, \mathrm{J})$, suggesting that the $\mathrm{CDK} 7 / \mathrm{CycH} / \mathrm{Mat} 1$ kinase complex is involved in modulating Yki-driven tissue growth. In contrast, knockdown of Xpd1 (Xpd1 ${ }^{\text {V106998; }}$ $X p d 1^{B L 65833}$ ), which recruits the CDK7/CycH/Mat1 complex to TFIIH, did not suppress eye overgrowth caused by $G M R>Y k i$ (Fig. 1M; Supplemental Fig. S1K,L), further supporting the notion that $\mathrm{CDK} 7$ can regulate Yki driven tissue growth independent of its role in basal transcription. Of note, knockdown of $\mathrm{CDK} 7, \mathrm{CycH}$, or Mat1 posterior to the morphogenetic furrow in otherwise wild-type eye imaginal discs where cells exit cell cycle and undergo differentiation, did not cause a discernible phenotype (Supplemental Fig. S1M-O), again suggesting that reduction in CDK7 activity does not affect its house-keeping function.

CDK7/CycH/Mat1 regulates Hippo pathway target genes and organ size independent of Wts

CDK7 RNAi in wing imaginal discs using hedgehog(hh)Gal4, which expressed Gal4 in the posterior compartment (Yue et al. 2012), diminished the expression of multiple Hippo pathway target genes including expanded lexlacZ), Diap1, and Wingless $(W g)$ as well as reduced the relative size of the posterior compartment in adult wings (Fig. 2A,B,E-G,J,K,N,O; Supplemental Fig. S2A-C'). Coexpression of CDK7 restored the expression of exlacZ in $h h>C D K 7^{V 10442}$ wing discs (Supplemental Fig. S2D-D"). Similarly, knockdown of either $\mathrm{CycH}$ (hh > $\left.C y c H^{V 104312}\right)$ or Mat1(hh>Mat1 $\left.{ }^{V 104780}\right)$ also diminished the expression of Hippo pathway target genes and reduced the relative size of the posterior compartment in adult wings (Fig. 2C-E, H,I,L,M,P,Q). Of note, the expression of $W g$ along the $\mathrm{D} / \mathrm{V}$ boundary, which is under the control of Notch signaling, was not affected by knockdown of any component of the CDK7-CycH-Mat1 complex (asterisks in Fig. 2O-Q, cf. anterior vs. posterior compartment), suggesting that inactivation of CDK7 selectively affects the Hippo pathway under our experimental conditions.

To determine where CDK7 acts in the Hippo pathway, we carried out genetic epistasis experiments. Knockdown of Wts in the P compartment of wing imaginal discs $(h h>$ $W t s^{V 106174}$ ) resulted in up-regulation of ex-lacZ and enlarged posterior compartment size (Fig. 2R; Supplemental Fig. S2E-E'I). Double knockdown of CDK7 and 

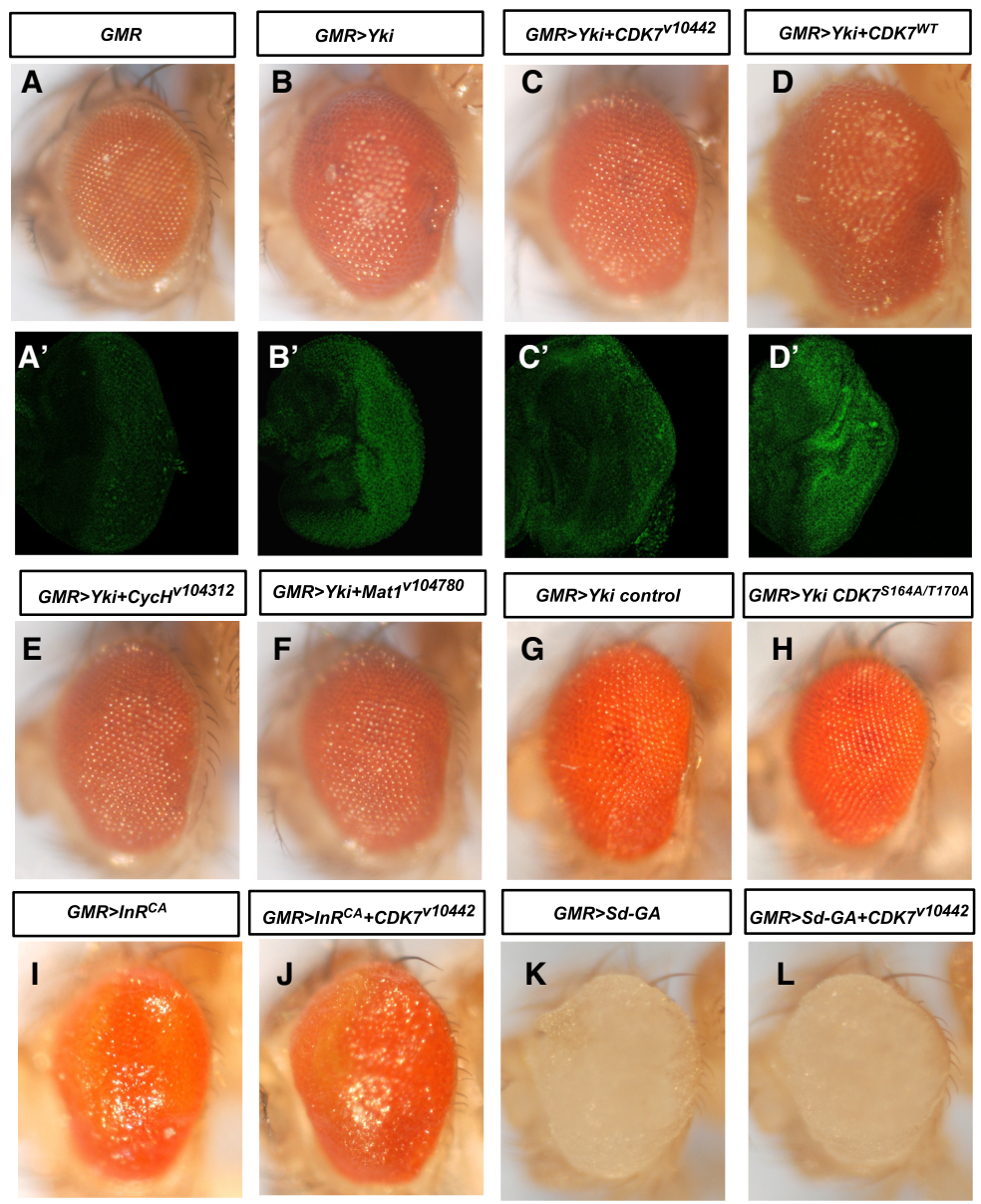

M

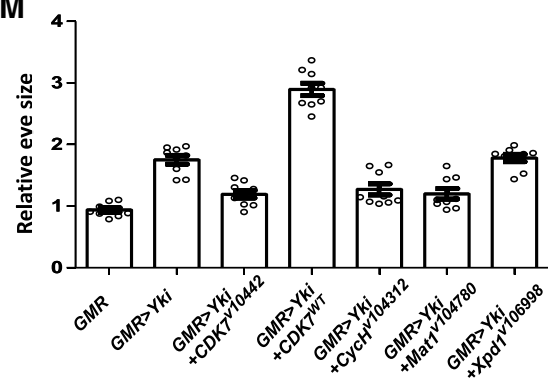

Wts $\left(h h>W t s^{V 106147}+C D K 7^{V 10442}\right)$ suppressed both the up-regulation of ex-lac $Z$ and overgrowth of the posterior compartment (Fig. 2S; Supplemental Fig. S2F-F'). In contrast, CDK7 RNAi did not affect the up-regulation of ex$l a c Z$ caused by $h h>S d-G A$ (Fig. 2T,U). In conjunction with the observation that CDK7 RNAi also failed to suppress Sd-GA-driven tissue overgrowth (Fig. 1K,L), these results suggest that CDK7 regulates Hippo signaling upstream of $S d$, but downstream from Wts.

Wts inhibits Yki activity by phosphorylating Yki on Ser111, Ser168, and Ser250 with S168 phosphorylation playing a more dominant role. Indeed, mutating Ser 168 $\left(\mathrm{Yki}^{\mathrm{S} 168 \mathrm{~A}}\right)$ dramatically increased $\mathrm{Yki}$ activity as indicated by the more dramatic overgrowth of eye caused by GMR > $Y k i^{S 168 A}$ compared with GMR $>Y k i^{W T}$ (Supplemental Fig.
S3A,C). Nevertheless, GMR $>Y k i^{S 168 A}$ driven eye overgrowth was suppressed by CDK7 RNAi (Supplemental Fig. S3B,D). Similarly, the eye overgrowth phenotype caused by $G M R>Y k i^{S 111 A}, S 250 A$ or $G M R>Y k i^{3 S A}$ (S111A, SS168A, S250A) was also reduced by CDK7 knockdown (Supplemental Fig. S3E-H). Hence, the activity of Wts-phosphorylation-deficient forms of Yki still depends on CDK7, consistent with the notion that CDK7 regulates Hippo signaling downstream from Wts.

\section{CDK7 regulates Yki stability in the nucleus}

The epistasis experiments conducted above suggest that CDK7 may regulate Hippo signaling at the level of Yki. Immunostaining of $h h>C D K 7^{V 10442}$ wing imaginal discs 


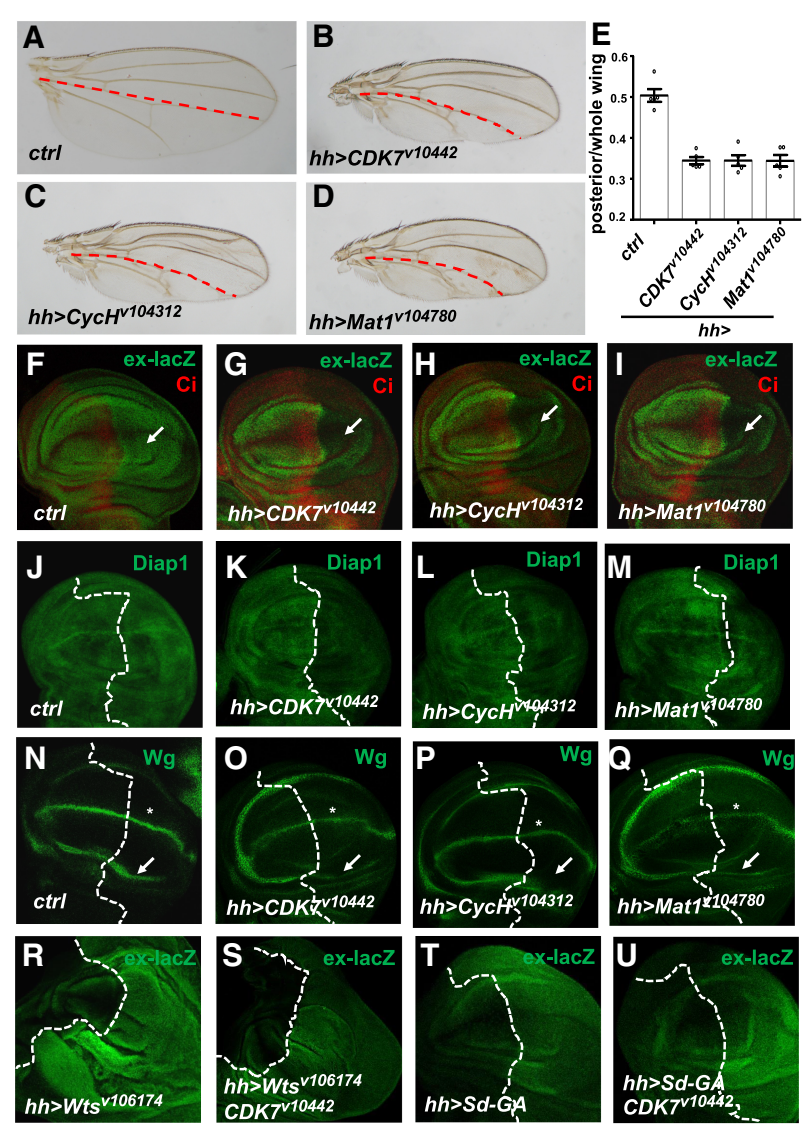

Figure 2. CDK7 regulates Hippo target gene expression downstream from Wts and upstream of Sd. $(A-D)$ Adult wings of hhGal4 (A) used as control (ctrl), hh-Gal4 UAS-CDK7-RNAi (B), hh-Gal4 UAS-CycH-RNAi (C), or hh-Gal4 UAS-Mat1-RNAi $(D)$. Dashed lines demarcate vein 4 of individual wings. Posterior is toward the bottom. (E) Quantification of posterior wing size (posterior to vein 4) relative to the size of entire wing. $(F-Q)$ Late third instar wing imaginal discs of hh-Gal4 (ctrl) $(F, J, N)$, hh-Gal4 UAS-CDK7-RNAi (G,K,O), hh-Gal4 UAS-CycH-RNAi $(H, L, P)$, or hh-Gal4 UAS-Mat1-RNAi $(I, M, Q)$ were immunostained to show the expression of Ci (red in $F-I$, not shown in $J-$ $Q$ ), and ex-lacZ (green in $F-I$ ), Diap1 (green in $J-M$ ), or Wg (green in $N-Q)$. Arrows in $F-I$ indicate $P$ compartments. Dashed lines in $J-Q$ demarcate the $\mathrm{D} / \mathrm{V}$ boundary based on $\mathrm{Ci}$ staining, which marks the anterior compartment. Arrows and asterisks in $N-Q$ indicate $\mathrm{Wg}$ expression at the periphery of the wing pouch region and along the $\mathrm{D} / \mathrm{V}$ boundary, respectively. $(R-U)$ Late third instar wing imaginal discs of hh-Gal4 UAS-Wts-RNAi (R), hh-Gal4 UAS-Wts-RNAi UAS-CDK7-RNAi (S), hh-Gal4 UAS-Sd-GA $(T)$, or hh-Gal4 UAS-Sd-GA UAS-CDK7-RNAi (U) were immunostained to show the expression of ex-lacZ. Dashed lines demarcate the $\mathrm{D} / \mathrm{V}$ boundary based on Ci staining (not shown).

with an anti-Yki antibody revealed that CDK7 RNAi reduced the level of endogenous Yki in P compartment cells (Fig. 3A-B'). Similar result was obtained by examination of a GFP knock-in Yki, Yki:GFP (Fig. 3C-D'; Fletcher et al. 2018). Expression of $C D K 7^{V 10442}$ under the control of a wing specific Gal4 driver MS1096 (MS >CDK $\left.7^{V 10442}\right)$ resulted in a reduction in overall wing size (Fig. 3E,F). While Yki mRNA level was not significantly altered in $M S$ >
$C D K 7^{V 10442}$ wing discs (Fig. 3G), Western blot analysis indicated that Yki protein level was down-regulated (Fig. $3 \mathrm{H})$, suggesting that $\mathrm{CDK} 7$ regulates Yki expression at the post-transcriptional level. CDK7 RNAi in S2 cells reduced the half-life of both transfected (Myc-Yki) and endogenous Yki (Fig. 3I,J), leading to diminished steadystate Yki protein expression (Fig. 3K,L). Treating S2 cells with the proteasome inhibitor MG132 not only increased the basal level of Yki but also restored the Yki level in CDK7 RNAi cells (Fig. 3K,L), suggesting that knockdown of CDK7 leads to Yki degradation through the ubiquitination-proteasome (UPS) pathway.

Because CDK7 is a nuclear kinase, we asked whether $\mathrm{CDK} 7$ regulates Yki stability in the nucleus. Fractionation of S2 cells transfected with Myc-Yki and treated with CDK7 dsRNA indicated that CDK7 RNAi reduced the level of nuclear Yki and to lesser extent, the level of cytoplasmic Yki (Fig. 3M, $M^{\prime}$ ). Preventing nuclear localization of Yki by addition of a myristoylation signal (Myr-Yki-GFP) made it insensitive to CDK7 RNAi (Fig. $3 N^{\prime}, N^{\prime}$ ), whereas forced nuclear localization of Yki by treating cells with a nuclear export inhibitor $\mathrm{LMB}$ resulted in reduction of nuclear but not cytoplasmic Yki (Fig. 30,0').

\section{CDK7 regulates Yki stability and activity by phosphorylating Yki S169}

The observations that CDK7 inactivation decreases nuclear Yki and Hippo target gene expression and that CDK7 acts downstream from Wts prompted us to determine whether CDK7 regulates Hippo signaling by directly phosphorylating Yki. CDK family kinases phosphorylate proline-based sites (S/T-P). Inspection of Yki protein sequence identified a total of six putative sites, among which only S169 is conserved between Yki and its mammalian homolog Yap and Taz (Fig. 4A). In an in vitro kinase assay, GST fusion protein containing an Yki fragment from aa160 to aa181 was phosphorylated by immunopurified CDK7 fused to GFP (GFP-CDK $7{ }^{\mathrm{WT}}$ ) but not by its kinase-dead version (GFP-CDK $7^{\mathrm{DR}}$ ) (Fig. 4B,C). Mutating $\mathrm{S} 169$ but not S168 or S172 to Ala abolished such phosphorylation (Fig. 4B,C), suggesting that CDK7 could phosphorylate YkiS169.

To further characterize Yki phosphorylation by CDK7, we generated a phospho-specific antibody for YkiS169, pS169 (see the Materials and Methods). In S2 cells, MycYki was phosphorylated on S169 detected by the pS169 antibody (Fig. 4D). The S169 phosphorylation signal of Myc-Yki was increased by cotransfection with wild-type $\left(\right.$ GFP-CDK $7{ }^{\mathrm{WT}}$ ) but not kinase-dead (GFP-CDK $7^{\mathrm{DR}}$ ) CDK7 (Fig. 4D). Mutating the S169 to Ala (Myc-Yki ${ }^{\text {S169A) }}$ abolished the phospho-signal, confirming the specificity of the pS169 antibody. The pS169 signal of Myr-Myc-Yki was not affected by GFP-CDK $7{ }^{\mathrm{WT}}$ (Fig. 4D), supporting that CDK7 phosphorylates YkiS169 in the nucleus. The pS169 antibody also detected the phosphorylation of endogenous Yki in S2 cells, which was enhanced by CDK7 transfection but diminished by CDK7 RNAi (Fig. 4E), suggesting that $\mathrm{CDK} 7$ is a major kinase responsible for YkiS169 phosphorylation of the endogenous Yki. 

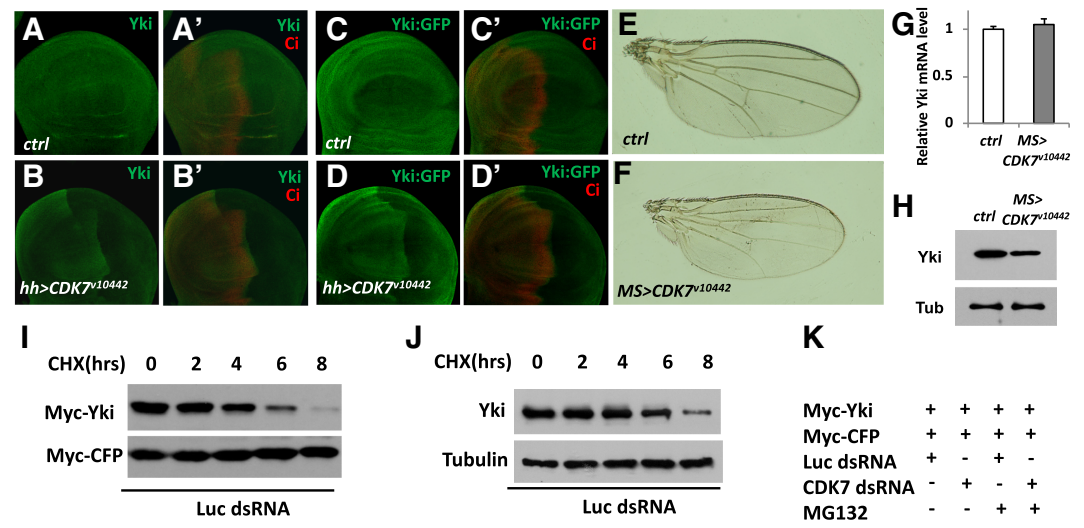

J

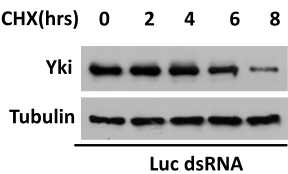

uc dSRNA

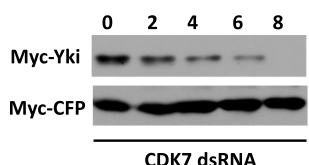

CDK7 dsRNA
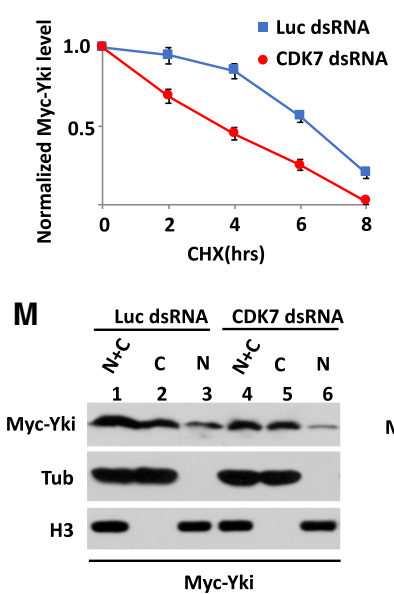

M'

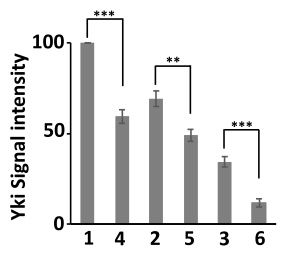

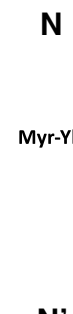

$\mathbf{N}$
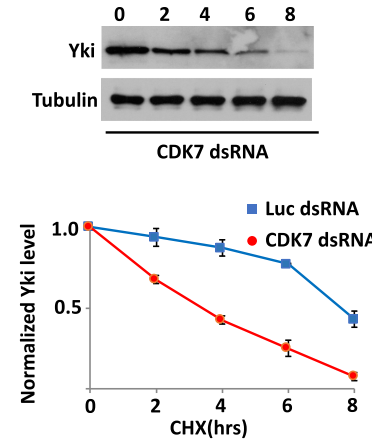

$\mathbf{N}$

Myr-Yki-G

Tub

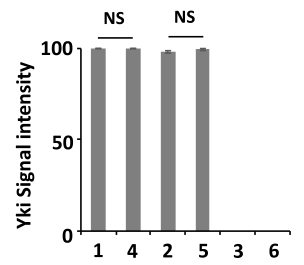

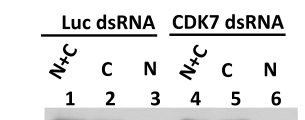

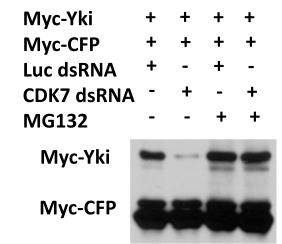

$\mathbf{L}$ $\begin{array}{llll}\text { CDK7 dsRNA }- & + & - & + \\ \text { MG132 } & - & - & +\end{array}$ Yki $-\quad$ Tubulin

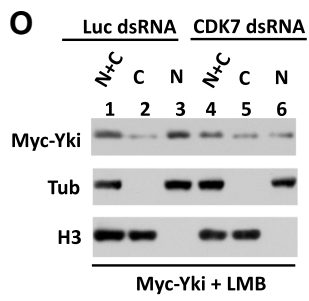

O'

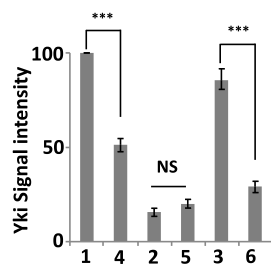

Luc dsRNA + - +

Figure 3. CDK7 regulates Yki stability in the nucleus. $\left(A-D^{\prime}\right)$ Late third instar wing imaginal discs of control $\left(A, A^{\prime}, C, C^{\prime}\right)$ or hh-Gal4 UASCDK7-RNAi $\left(B, B^{\prime}, D, D^{\prime}\right)$ were immunostained to show the expression of $\mathrm{Ci}$ (red in $A^{\prime}-C^{\prime}$ ) and Yki (green in $A-B^{\prime}$ ) or Yki:GFP (green in $\left.C-D^{\prime}\right) \cdot(E, F)$ Control adult wing $(E)$ or adult wing expressing UAS-CDK7-RNAi under the control of MS1096 Gal4 driver $(F) .(G)$ Relative Yki mRNA expression determined by RTqPCR in wing discs of the indicated genotypes. $(H)$ Western blot analysis of Yki protein levels in wing discs of the indicated genotypes. $(I, J)$ Western blot analysis and quantification of Myc-Yki $(I)$ or endogenous Yki $(J)$ in S2 cells transfected with luciferase (Luc) dsRNA or CDK7 dsRNA at the indicated time point following cycloheximide (CHX) treatment. MycCFP was used as loading control for Myc-Yki. $(K, L)$ Western blot analysis of Myc-Yki $(K)$ or endogenous Yki $(L)$ in S2 cells transfected with Luc dsRNA or CDK7 dsRNA and treated with or without MG132 for 4 h. $\left(M-O^{\prime}\right)$ Western blot analysis $(M-O)$ and quantification $\left(M^{\prime}-O^{\prime}\right)$ of Myc-Yki, or Myr-Yki-GFP in whole-cell lysates $(\mathrm{N}+\mathrm{C})$, cytoplasmic fraction $(\mathrm{C})$, or nuclear fraction $(\mathrm{N})$ from $\mathrm{S} 2$ cells transfected with Luc dsRNA or CDK7 dsRNA. In $O$, cells were treated with $20 \mathrm{nM} \mathrm{LMB}$ for $4 \mathrm{~h}$ before harvesting. Data are mean $\pm \mathrm{SD}$. (NS) not significant; $\left.\left.{ }^{* *}\right) P<0.01 ;{ }^{* * *}\right) P<0.001$ (Student's $t$-test).

When expressed in S2 cells, the steady-state level of Myc-Yki was decreased by S169A mutation (MycYki ${ }^{\text {S169A) }}$ but increased by S169D mutation (Myc-

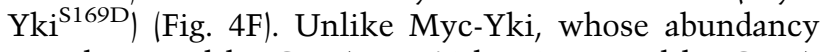
was decreased by CDK7 RNAi but increased by CDK7 cotransfection, neither Myc-Yki ${ }^{\mathrm{S} 169 \mathrm{~A}}$ nor Myc-Yki ${ }^{\mathrm{S} 169 \mathrm{D}}$ was responsive to CDK7 RNAi or overexpression (Fig. 4F). In a Sd-luc reporter assay (Zhang et al. 2008), Myc$\mathrm{Yki}^{\text {S169A }}$ exhibited lower, while Myc-Yki ${ }^{\text {S169D }}$ exhibited higher transcriptional activity than Myc-Yki ${ }^{\mathrm{WT}}$, and the activity of neither Myc-Yki ${ }^{\text {S169A }}$ nor Myc-Yki ${ }^{\text {S169D }}$ was affected by CDK7 RNAi (Fig. 4G).

To determine whether CDK7 regulates Yki activity and organ size by phosphorylating YkiS169 in vivo, we generated transgenic flies expressing UAS-Yki ${ }^{S 169 A}$ or UAS$Y k i^{S 169 D}$ under the control of GMR-Gal4. We found that

$\mathrm{Yki}^{\mathrm{S} 169 \mathrm{D}}$ was more potent, while $\mathrm{Yki}{ }^{\mathrm{S} 169 \mathrm{~A}}$ less potent, in driving eye overgrowth compared with $\mathrm{Yki}^{\mathrm{WT}}$ (Fig. 4H-J, Q). Unlike $G M R>Y k i^{W T}$ whose phenotype was modulated by altering CDK7 activity, the eye overgrowth phenotype caused by GMR $-Y k i^{S 169 A}$ or GMR-Yki ${ }^{S 169 D}$ was no longer modified by either RNAi or overexpression of CDK7 (Fig. 4K-Q). Taken together, these results demonstrate that CDK7 promotes Yki stability and activity by phosphorylating YkiS169.

\section{Yki is degraded by CRL4 $4^{D C A F 12}$ in the absence of CDK7}

The above results suggest that nuclear Yki is intrinsically unstable but phosphorylation by CDK7 on S169 increases its half-life and thus sustains the Hippo pathway output. Because the proteasome inhibitor MG132 could stabilize 
A

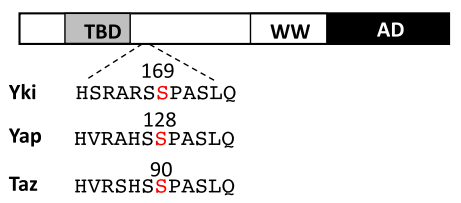

B

$\begin{array}{lc}\begin{array}{l}\text { GST-Yki } \\ \text { fusion Protein }\end{array} & \text { Yki fragment 160-181 } \\ & 169 \\ \text { GST-Yki-WT } & \text { AIHHSRARSSPASLQQNYNVRA } \\ \text { GST-Yki-S168A } & \text { AIHHSRARASPASLQQNYNVRA } \\ \text { GST-Yki-S169A } & \text { AIHHSRARSAPASLQQNYNVRA } \\ \text { GST-Yki-S172A } & \text { AIHHSRARSSPAALQQNYNVRA }\end{array}$

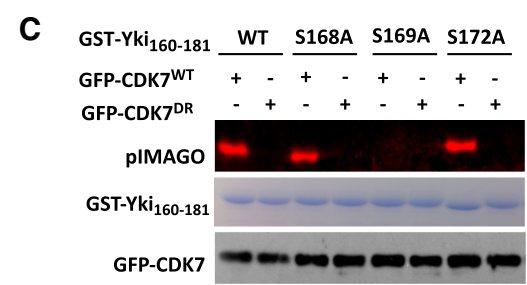

E

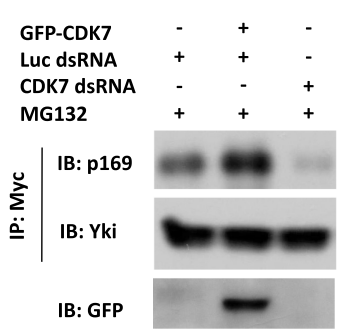

G
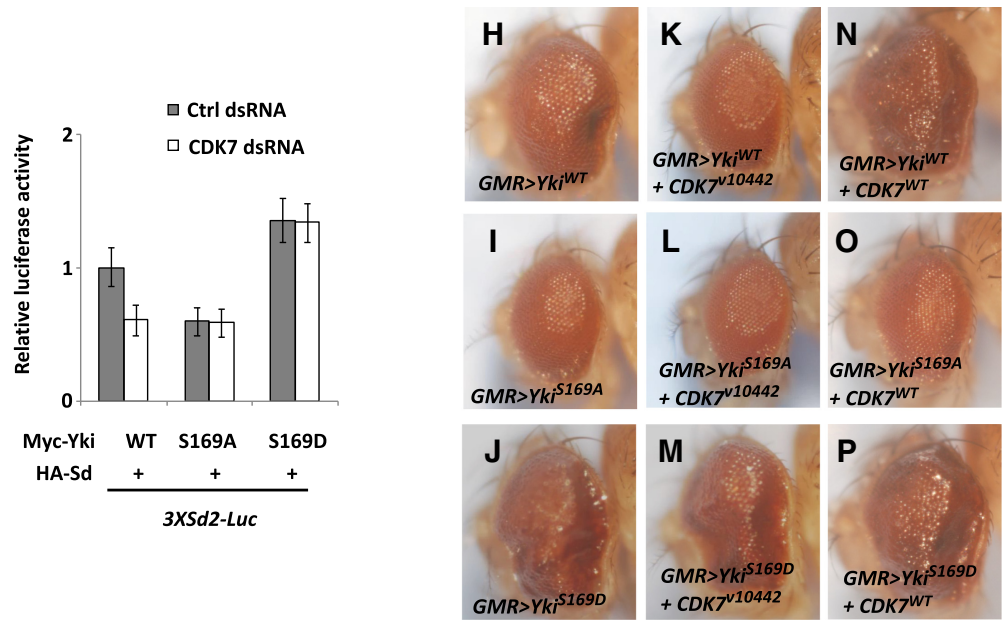

$\mathbf{F}$
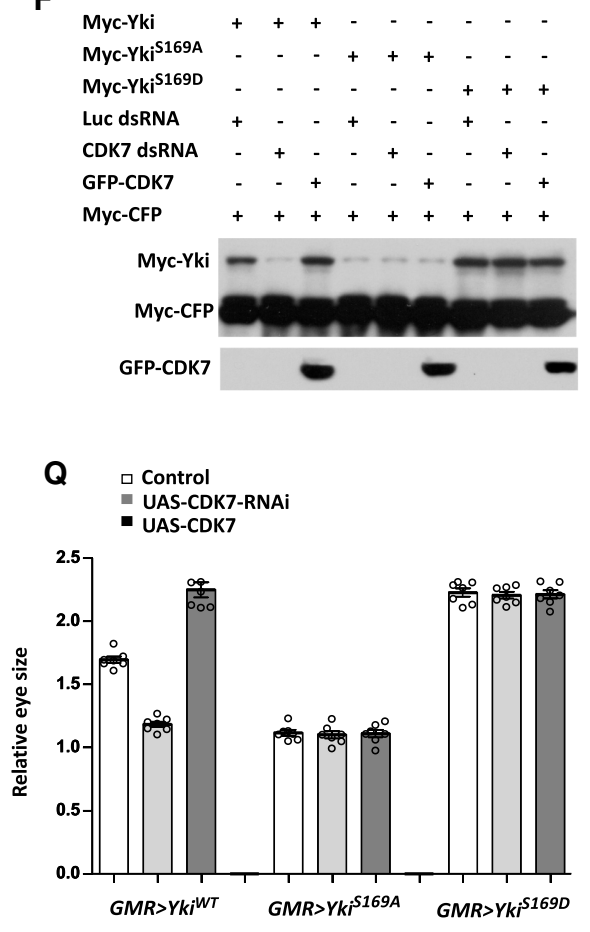

Figure 4. CDK7 regulates Yki stability and Hippo signaling by phosphorylating YkiS169. (A) Diagram of Yki protein with the amino acid sequences surround YkiS169, YapS128, and TazS90 shown underneath. TBD, WW, and AD indicate the TEAD binding domain, WW domain, and activation domain of Yki, respectively. (B) GST-Yki fusion proteins containing the indicated wild-type or mutated Yki sequences. $(C)$ In vitro kinase assay using immunopurified wild-type (WT) or kinase-dead (DR) GFP-CDK7 as kinase and the indicated GST-Yki fusion proteins as substrates. $(D)$ Western blot analysis to examine S169 phosphorylation of the indicated Myc-Yki proteins expressed in S2 cells cotransfected with or without wild-type (WT) or kinase-dead (DR) GFP-CDK7 and treated with MG132. (E) S169 phosphorylation of the endogenous Yki in S2 cells transfected with or without wild-type GFP-CDK7, Luc dsRNA, or CDK7 dsRNA. (F) Western blot analysis of the indicated Myc-Yki proteins expressed in S2 cells cotransfected with Myc-CFP and with or without wild-type GFP-CDK7, Luc dsRNA or CDK7 dsRNA. (G) 3XSd2-luc reporter assay in S2 cells expressing the indicated Myc-Yki constructs and HA-Sd. $(H-Q)$ Side view of Drosophila adult eyes $(H-P)$ and quantification of the size of adult eyes $(Q)$ of the indicated genotypes.

Yki in CDK7 knockdown cells (Fig. 3K,L), nuclear Yki is degraded by the UPS pathway. To identify the E3 ubiquitin ligase(s) responsible for Yki degradation in the absence of CDK7, we carried out a genetic modifier screen using transgenic RNAi lines targeting Drosophila E3 ubiquitin ligases including the Cullin family of modular E3 ubiquitin ligases. We identified two independent UAS-Cul4RNAi lines (v105668, v44829) that enhanced the eye overgrowth phenotype caused by GMR-Yki (Fig. 5A,B). Cul4 forms a multisubunit E3 ubiquitin ligase (CRL4) complex in which the DNA-damage binding protein 1 (DDB1) bridges Cul4 to multiple DDB1-Cul4-associated factors
(DCAFs) that serves as substrate receptors (Angers et al. 2006; Lee and Zhou 2007). We therefore screened RNAi lines that target Drosophila DCAFs and identified a UAS-DCAF12-RNAi line (v43758) that enhanced the GMR-Yki phenotype similar to UAS-Cul4-RNAi (Fig. $5 C)$. In addition, we found that DCAF12 RNAi could rescue the phenotypes caused by CDK7 RNAi. For example, the suppression of GMR-Yki driven eye overgrowth by CDK7 RNAi was negated by DCAF12 RNAi (Fig. 5D-F). Expression of UAS-CDK7-RNAi using eyeless-Gal4 (ey $>C D K 7^{v 10442}$ ) that drives $U A S$ transgene expression in eye imaginal disc at a much earlier stage than GMR-gal4 

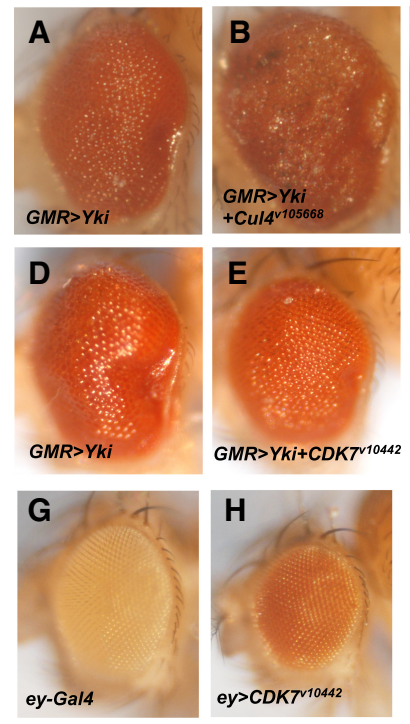

Q
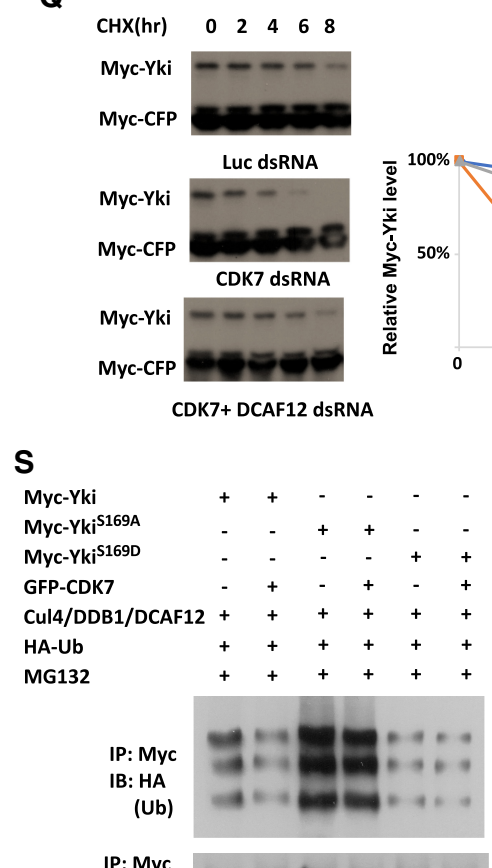

B: Myc
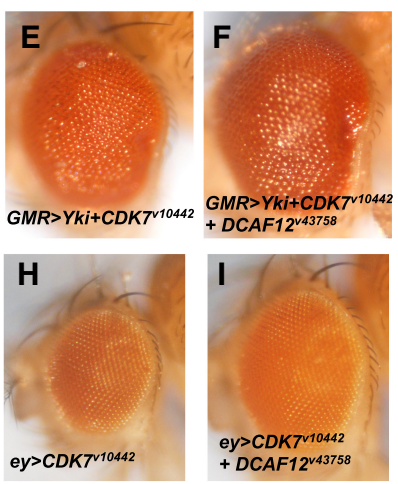

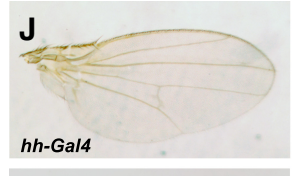

L $h$ h $>$ DCAF12 $2^{\text {143758 }}$
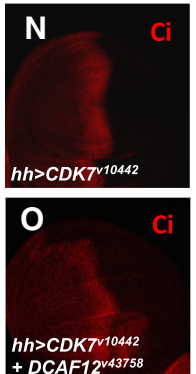

+ DCAF12 $^{\text {va3758 }}$
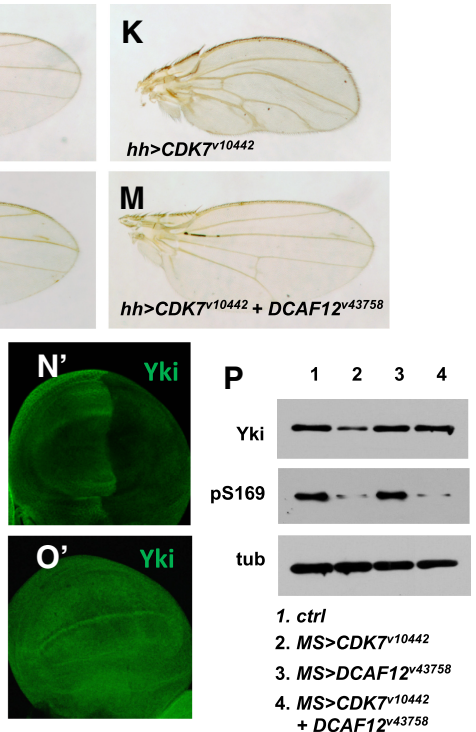

R

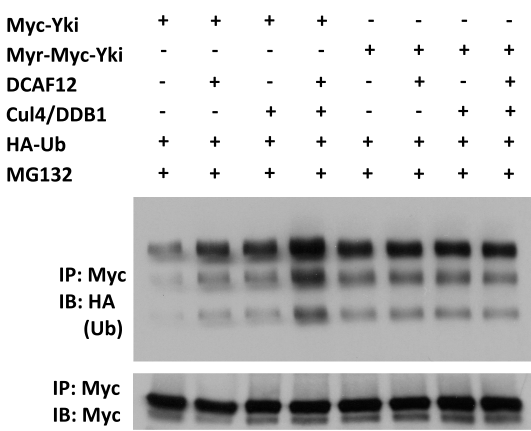

$\mathbf{T}$

Myc-Yki Luc dsRNA CDK7 dsRNA DCAF12 dsRNA Cul4/DDB1 dsRNA HA-Ub MG132
Luc dsRNA

- CDK7 dsRNA - CDK7/DCAF12 dsRNA

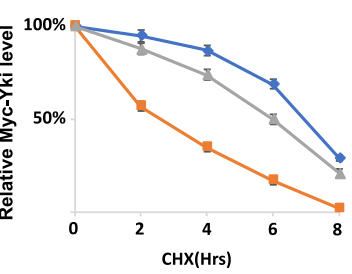

IB: Myc

U
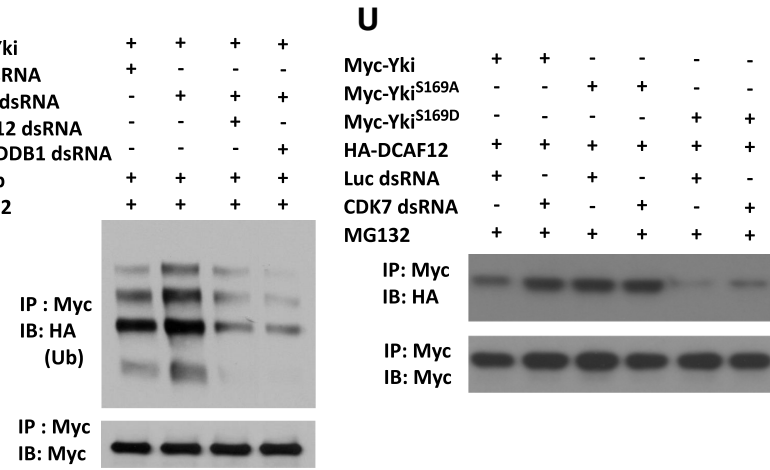

Figure 5. Yki is targeted for degradation by the CRL4 ${ }^{\mathrm{DCAF} 12}$ ubiquitin ligase. $(A-I)$ Side view of adult eyes of the indicated genotypes. $(J-M)$ Adult wings of the indicated genotypes. $\left(N-\mathrm{O}^{\prime}\right)$ Late third instar wing imaginal discs of the indicated genotypes were immunostained to show the expression of $\mathrm{Ci}$ (red) and Yki (green). (P) Western blot analysis of late third instar wing discs of the indicated genotypes with Yki, pS169, and tubulin (tub) antibodies. (Q) Western blot analysis of Myc-Yki level in S2 cells transfected with luciferase (Luc) dsRNA (top), CDK7 dsRNA (middle), or CDK7 and DCAF12 dsRNAs (bottom) at the indicated time point following cycloheximide (CHX) treatment. Myc-CFP was used as loading control. Quantification of Myc-Yki at the indicated time point following cycloheximide (CHX) treatment is shown at the right. $(R)$ Ubiquitination of Myc-Yki or Myr-Myc-Yki in S2 cells cotransfected with DCAF12, DDB1/Cul4, or DCAF12/DDB1/Cul4. (S) Ubiquitination of Myc-Yki, Myc-Yki ${ }^{\text {S169A }}$, or Myc-Yki ${ }^{\text {S169D }}$ in S2 cells cotransfected with DCAF12/DDB1/ Cul4 and with or without GFP-CDK7. (T) Ubiquitination of Myc-Yki in S2 cells transfected with Luc dsRNA, CDK7 dsRNA, CDK7/ DCAF12 dsRNAs, or CDK7/DDB1/Cul4 dsRNAs and treated with or without MG132. (U) Western blot analysis of HA-DCAF12 coimmunoprecipitated with Myc-Yki, Myc-YkiS ${ }^{169 A}$ or Myc-YkiS ${ }^{169 D}$ from S2 cells transfected with Luc or CDK7 dsRNA and treated with MG132. 
(Halder et al. 1998) reduced eye growth (Fig. 5G,H), which was rescued by simultaneous knockdown of DCAF12 $\left(\right.$ ey $\left.>C D K 7^{V 10442}+D C A F 12^{v 43758}\right)$ (Fig. 5I). Similarly, the reduced posterior wing size caused by $h h>C D K 7^{V 10442}$ was restored to normal size by DCAF12 RNAi even though DCAF12 RNAi alone did not affect the wing size (Fig. 5J-M). DCAF12 RNAi restored Yki to normal levels in CDK7 knockdown wing discs as determined by both immunostaining and Western blot analysis (Fig. 5N-P). In S2 cells, the reduction in Myc-Yki half-life caused by CDK7 RNAi was also rescued by DCAF12 RNAi (Fig. $5 \mathrm{Q})$. Taken together, these results demonstrate that Yki was degraded by CRL4 ${ }^{\mathrm{DCAF} 12}$ when CDK7 was inactivated. Of note, although Yki protein level was restored to the wild-type level in DCAF12 and CDK7 double RNAi wing discs compared with CDK7 single RNAi wing discs, the pS169 signal remained lower than that of wild-type or DCAF12 single RNAi wing discs (Fig. 5P), suggesting that CDK7 is responsible for phosphorylating endogenous Yki at S169 in vivo.

We then used a cell-based assay to determine whether CRL4 ${ }^{\text {DCAF12 }}$ catalyzed the ubiquitination of Yki and whether CRL4 ${ }^{\text {DCAF12 }}$-mediated ubiquitination of Yki is regulated by CDK7. In S2 cells, Myc-Yki ubiquitination was promoted by coexpressed DCAF12, DDB1, and Cul4 (Fig. 5R). In contrast, CRL4 ${ }^{\text {DCAF12 }}$ did not promote the ubiquitination of the membrane-tethered Yki (MyrMyc-Yki; Fig. 5R), consistent with a previous finding DCAF12 forms a nuclear complex with Cul4 and thus targets nuclear proteins for ubiquitination and degradation (Patrón et al. 2019). CRL4 ${ }^{\text {DCAF12 }}$-mediated Myc-Yki ubiquitination was inhibited by coexpression of CDK7 or by the S169D mutation but increased by the S169A mutation or by CDK7 RNAi (Fig. 5S,T). In addition, knockdown of DCAF12 or Cul4/DDB1 suppressed Myc-Yki ubiquitination in CDK7-depleted cells (Fig. 5T).

Because the DCAF proteins are the substrate recognition components of CRL4 E3 ligase complexes, we asked whether DCAF12 interacts with Yki in a manner regulated by CDK7. CoIP experiments showed that HA-DCAF12 interacted with Myc-Yki and this interaction was enhanced by CDK7 RNAi (Fig. 5U). S169A increased whereas S169D decreased the interaction of the corresponding Yki mutants (Myc-Yki ${ }^{\text {S169A }}$ and Myc-Yki ${ }^{\text {S169D }}$ ) with HADCAF12 (Fig. 5U). Taken together, these results suggest that DCAF12 binds Yki and recruits DDB1-Cul4 to ubiquitinate Yki in the absence of CDK7 and that CDK7-mediated phosphorylation of YkiS169 inhibits the recruitment of CRL4 ${ }^{\mathrm{DCAF} 12}$ and thus the ubiquitination of Yki, leading to Yki stabilization in the nucleus.

\section{CDK7 stabilizes Yap/Taz by inhibiting DCAF12- mediated ubiquitination and degradation}

We next determined whether CDK7 played a conserved role in the regulation of Yap/Taz stability. CDK7 RNAi resulted in a reduction of Yap/Taz level in both wild-type and Lats1/2 KO HEK293 cells (Fig. 6A,B), suggesting that CDK7 promotes Yap/Taz stabilization in a manner independent of Lats1/2, which is consistent with the finding in Drosophila. Serum depletion of HEK293 cells, which caused nuclear exclusion of Yap/Taz (Meng et al. 2015; Cho et al. 2018), made Yap/Taz less sensitive to CDK7 inactivation (Supplemental Fig. S4A). Fractionation of serum-depleted and control HEK293 cells revealed that CDK7 RNAi caused a reduction of Yap/Taz mainly in the nuclear fraction (Supplemental Fig. S4B,C), suggesting that CDK7 regulates the stability of nuclear Yap/Taz.

Western blot analysis using a phospho-specific antibody that recognized phosphorylated YapS128 (pS128) (Moon et al. 2017) revealed that overexpression of CDK7 promoted phosphorylation of coexpressed Flag-tagged Yap (Fg-Yap), whereas CDK7 RNAi inhibited S128 phosphorylation of both Fg-Yap and endogenous Yap in HEK293 cells (Fig. 6C-E). To determine the effect of S128 phosphorylation on Yap stability and activity, we generated Flag-tagged S128A and S128D Yap variants (Fg-Yap ${ }^{\text {S128A }}$ and Fg-Yap $\left.{ }^{\mathrm{S} 128 \mathrm{D}}\right)$. When expressed in HEK293 cells, Fg-Yap ${ }^{\text {S128A }}$ exhibited reduced, whereas Fg-Yap ${ }^{\text {S128D }}$ exhibited increased steady-state levels and transcriptional activity compared with wild-type Fg-Yap (Fig. 6F,G). Furthermore, both the protein level and transcriptional activity of either $\mathrm{Yap}^{\mathrm{S} 128 \mathrm{~A}}$ or $\mathrm{Yap}^{\mathrm{S} 128 \mathrm{D}}$ were no longer affected by CDK7 RNAi (Fig. 6F,G). TazS90 is equivalent to YapS128 (Fig. 4A). We found that S90A decreased, whereas S90D increased, Taz protein stability and transcriptional activity in HEK293 cells (Fig. 6H,I). In addition, these mutations rendered Taz insensitive to CDK7 inactivation (Fig. 6H,I). Taken together, these results suggest that CDK7 promotes the stability and activity of Yap/Taz by phosphorylating YapS128/TazS90.

To determine whether DCAF12 is responsible for Yap/ Taz ubiquitination and degradation when CDK7 is inactivated, we knocked down DCAF12 together with CDK7 by siRNA in HEK293 cells. DCAF12 RNAi increased the steady state level of Yap/Taz and suppressed the downregulation of Yap/Taz caused by CDK7 RNAi (Fig. 6J,K). CDK7 RNAi increased Yap/Taz ubiquitination, whereas DCAF12 RNAi inhibited both basal and CDK7 RNAi-induced ubiquitination of Yap/Taz (Fig. 6L,M). CDK7 RNAi increased the association of Yap/Taz with DCAF12 (Fig. $6 \mathrm{~N}, \mathrm{O})$. The S128A/S90A mutation increased, whereas the S128D/S90D mutation decreased the binding of DCAF12 to Yap/Taz (Fig. 6N,O) as well as ubiquitination of Yap/Taz (Fig. 6P,Q). Taken together, these results demonstrate that DCAF12 mediates the ubiquitination and degradation of Yap/Taz and that CDK7-mediated phosphorylation of YapS128/TazS90 inhibits the binding of DCAF12, thus blocking the ubiquitination and degradation of Yap/Taz.

\section{CDK7 inhibition opposes Yap/Taz-driven cancer cell growth}

Yap and Taz have been implicated as oncoproteins in a wide range of human cancer including triple negative breast cancer (TNBC), colorectal cancer (CRC), and esophageal cancer (Zanconato et al. 2016b). In support of this, inactivation of Yap/Taz affected the growth of TNBC cell line MDA-MB-231, CRC cell line HCT116, and 


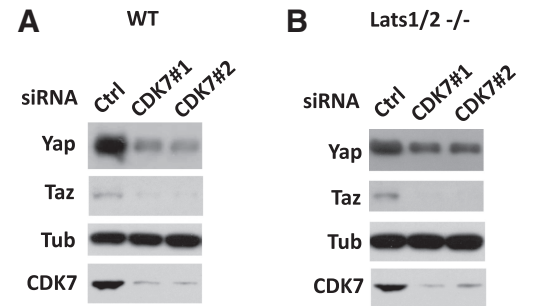

F

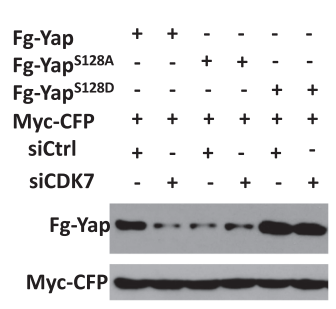

$\mathbf{J}$

N
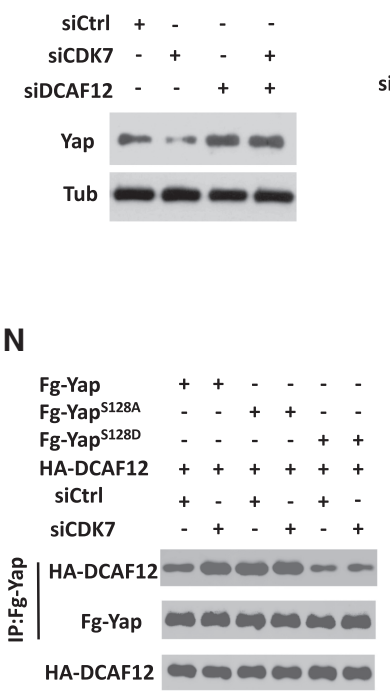

K

0
C

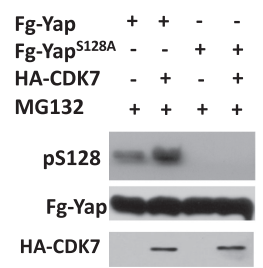

H
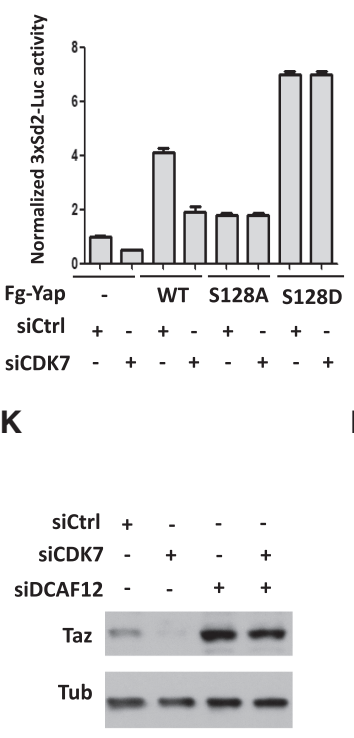

$\mathbf{L}$
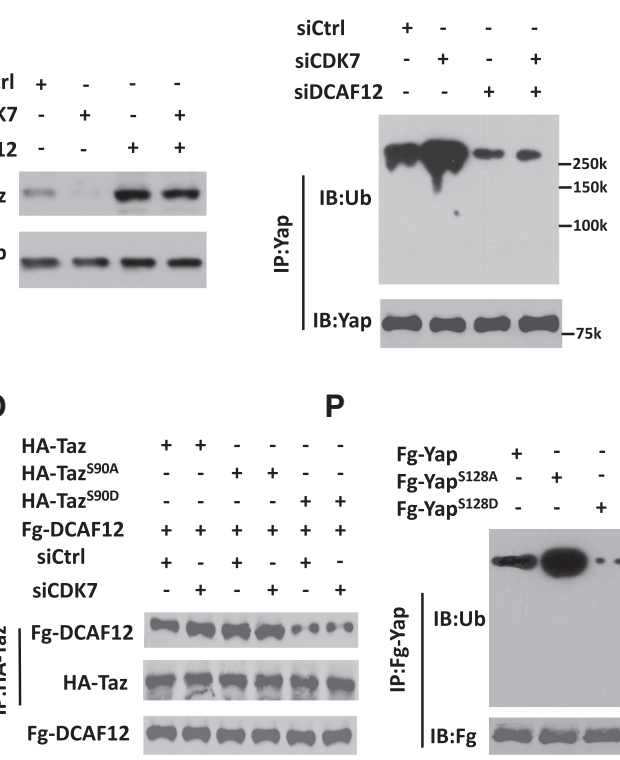

D

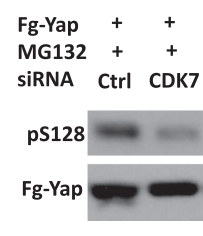

E

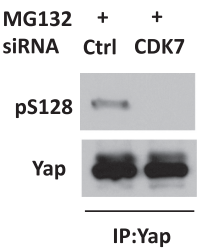

I

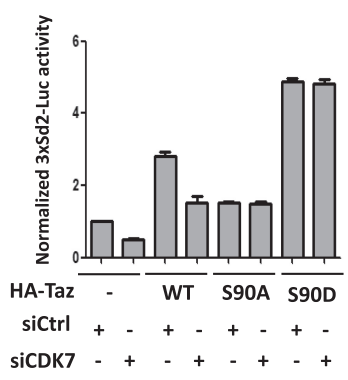

M

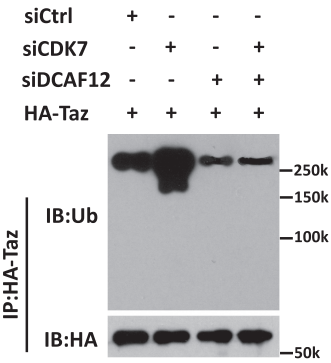

Q

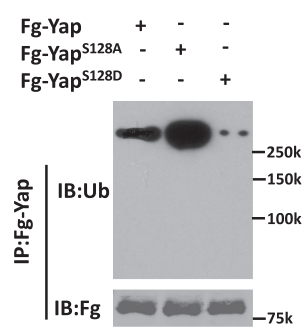

$\begin{array}{llll}\text { HA-Taz } & + & - & - \\ \text { HA-Taz }^{590 A} & - & + & -\end{array}$

$\mathrm{HA}-T a z^{\text {S9OD }}-$ -

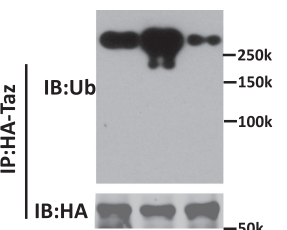

Figure 6. CDK7 plays conserved role in the regulation of Yap/Taz and Hippo signaling. $(A, B)$ Western blot analysis of Yap/Taz in wildtype $(A)$ or Lats $1 / 2^{-/-}$HEK293 cells treated with control or CDK7 siRNA. $(C, D)$ S128 phosphorylation of Fg-Yap in HEK293 cells with or without cotransfection with HA-CDK7 $(C)$ or treated with control or CDK7 siRNA $(D)$. $(E)$ S128 phosphorylation of endogenous Yap in HEK293 cells treated with control or CDK7 siRNA. $(F, G)$ Western blot analysis of Fg-Yki $(F)$ or 3xSd-Luc reporter assay $(G)$ of HEK293 cells transfected with the indicated Fg-Yki constructs and treated with control or CDK7 siRNA. $(H, I)$ Western blot analysis of HA-Taz $(H)$ or 3xSd-Luc reporter assay $(I)$ of HEK293 cells transfected with the indicated HA-Taz constructs and treated with control or CDK7 siRNA. $(J, K)$ Western blot analysis of Yap $(J)$ or Taz $(K)$ in HEK293 cells treated with the indicated siRNAs. $(L, M)$ Ubiquitination of Yap $(L)$ or HA-Taz $(M)$ in HEK293 cells treated with the indicated siRNAs. $(N, O)$ Coimmunoprecipitation experiments to detect interaction between HA-DCAF12 with Fg-Yap and its variants $(N)$ or Fg-DCAF12 with HA-Taz and its variants $(O)$ in HEK293 cells transfected with the indicated constructs and treated with control or CDK7 siRNA. $(P, Q)$ Ubiquitination of Fg-Yap and its variants $(P)$ or HA-Taz and its variants $(Q)$ in HEK293 cells.

esophagus cancer line EC9706 (Mo et al. 2012; Zanconato et al. 2015; Chang et al. 2017). We found that CDK7 knockdown by siRNAs reduced Yap level, Hippo target gene expression, cell growth, and invasiveness of these cancer cell lines (Supplemental Fig. S5). Exogenous ex- pression of a wild-type Yap partially rescued, whereas expression of Yap ${ }^{\mathrm{SD} 128}$ more completely rescued Yap protein level, Hippo target gene expression, cell growth, and invasiveness of CDK7-depleted MDA-MB-231 cells (Fig. 7AE), suggesting that CDK7 promotes cancer cell growth 
A

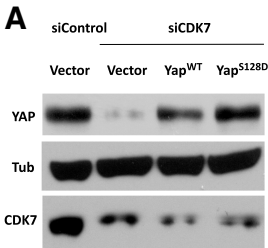

B

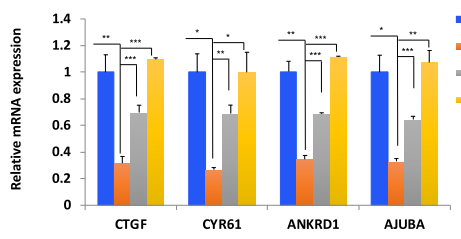

D
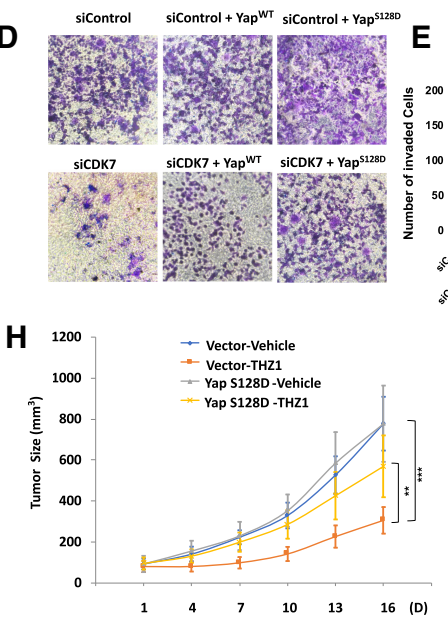

K
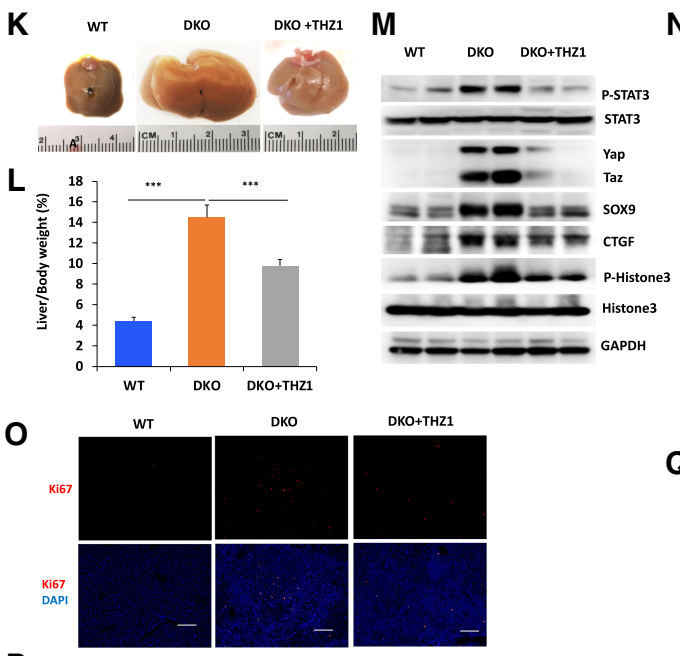

DKO+THZ1

P

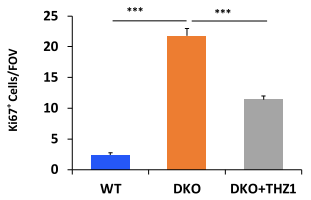

F

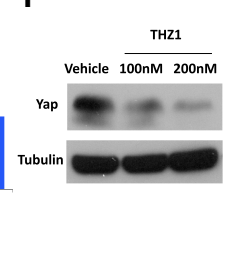

I
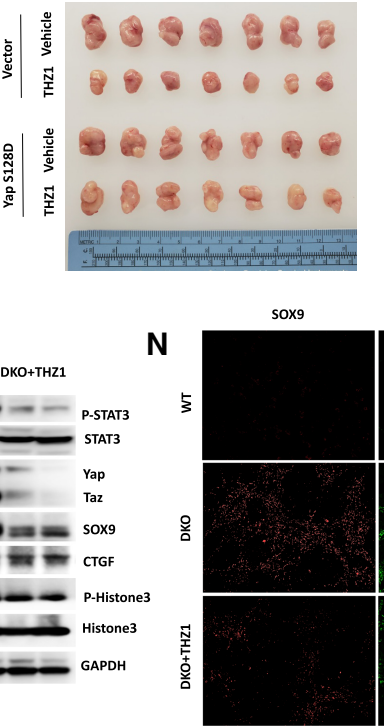

DAPI

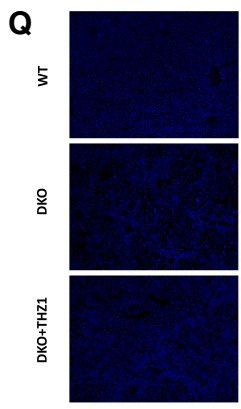

C

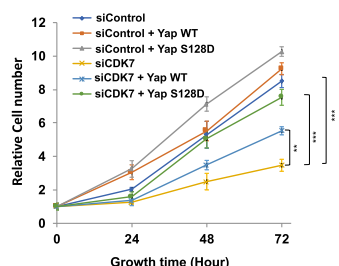

G

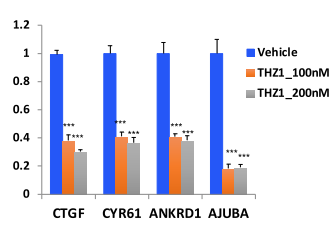

J

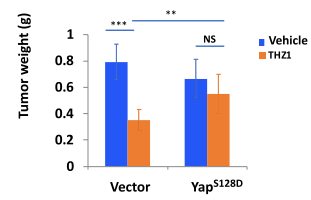

EPCAM

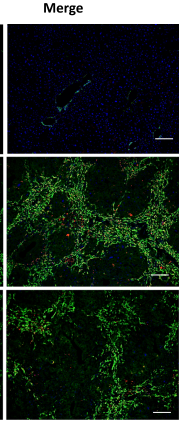

F4/80

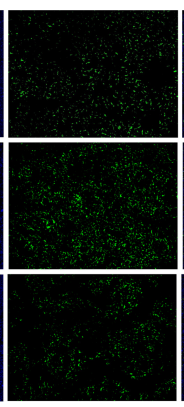

Merge

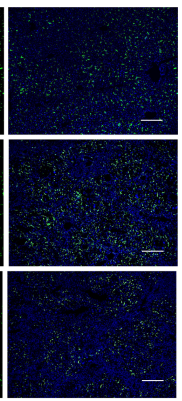

Figure 7. CDK7 regulates organ size and tumor progression through Yap/Taz. (A) Western blot analysis of Yap and CDK7 in MDA-MB231 cells transfected with siControl or siCDK7 and infected with lentiviruses expressing the empty vector, wild-type Yap, or the S128D mutant. $(B)$ Relative mRNA levels of the indicated Hippo target genes in the indicated MDA-MB-231 cells were measured by RT-qPCR. Data are means \pm SD. $\left.\left.\left(^{*}\right) P<0.05 ;{ }^{* *}\right) P<0.01 ;{ }^{* * *}\right) P<0.001$ (Student's $t$-test). $(C)$ Relative cell numbers of MDA-MB-231 cells treated with siControl or siCDK7 and infected with lentiviruses expressing wild-type or S128D Yap were measured by the WST-1 assay at different time points after infection. Data are means \pm SD. $\left(^{* *}\right) P<0.01 ;\left({ }^{* *}\right) P<0.001$ (Student's $t$-test). $(D, E)$ Transwell invasion assay of MDAMB-231 cells treated with siControl or siCDK7 and infected with lentiviruses expressing wild-type or S128D Yap. Data are means \pm SD. $\left({ }^{* *}\right) P<0.01 ;\left({ }^{* * *}\right) P<0.001$ (Student's $t$-test). $(F)$ Western blot analysis of Yap in MDA-MB-231 cells treated with THZ1. $(G)$ Relative mRNA levels of the indicated Hippo target genes in MDA-MB-231 cells treated with THZ1. Data are means \pm SD. $\left(^{* * *}\right) P<$ 0.001 (Student's $t$-test). (H) Tumor size of the indicated MDA-MB-231 xenografts in mice treated with vehicle or THZ1 at the indicated time after drug treatment. (I) Macroscopic images of tumors derived from the indicated MDA-MB-231 xenografts at the end of drug treatment. $(J)$ Quantification of tumor weight of the indicated MDA-MB-231 xenografts at the end of drug treatment. Data are means \pm SD. $n=7$ mice for each group. (NS) not significant; $\left.{ }^{* *}\right) P<0.01$; $\left.{ }^{* * *}\right) P<0.001$ (Student's $t$-test). $(K) \mathrm{MST} 1 / 2$ DKO mice were treated with vehicle or THZ1 $(10 \mathrm{mg} / \mathrm{kg})$ every other day starting at the age of $1 \mathrm{mo}$ until 3 mo old. Representative macroscopic images of livers were shown. $(L)$ Quantitative analysis of liver-to-body weight ratio. Data are means \pm SD. $n=6$ mice for each group. $\left(^{* * *}\right) P<0.001$ (Student's $t$-test). $(M)$ Western blot analysis of control and THZ1-treated liver tissues of the indicated genotypes using the indicated antibodies. ( $N, O, Q)$ Liver cross-sections of the indicated genotypes treated with vehicle or THZ1 were subjected to immunostaining with anti-EPCAM, SOX9, F4/ 80, or Ki67 antibody. Scale bar, $100 \mu \mathrm{m}$. (P) Quantification of Ki67 ${ }^{+}$cells in liver sections of the indicated genotypes treated with vehicle or THZ1. Data are means \pm SD. $n=5$ sections for each group. $\left.{ }^{* * *}\right) P<0.001$ (Student's $t$-test). 
and invasiveness, at least in part, through stabilizing Yap. Of note, overexpression of Yap ${ }^{\mathrm{WT}}$ or $\mathrm{YapS}^{128 \mathrm{D}}$ in control MDA-MB-231 cells only slightly increased their growth and invasiveness (Fig. 7C-E) because MDA-MB-231 cells already have high Yap/Taz activity due to their loss of NF2 (Zanconato et al. 2015). Treating MDA-MB-231 cells with a CDK7 pharmacological inhibitor THZ1 also reduced Yap protein level and Hippo target gene expression (Fig. 7F,G).

To determine whether CDK7 promotes tumor growth through Yap in vivo, we generated xenograft models of TNBC by transplanted MDA-MB-231 cells expressing pLVX-IRES-ZSgreen1 (vector) or pLVX-IRES-ZSgreen1Yki-S128D (YAP ${ }^{\text {S128D) }}$ ) into the mammary fat pads of nude mice. After tumors reached $\sim 100 \mathrm{~mm}^{3}$, mice were treated with vehicle or THZ1 (10 mg/kg twice a day) continuously for $16 \mathrm{~d}$. Tumor size was measured every $3 \mathrm{~d}$ during treatment and tumors were weighed at the end of treatment. Compared with vehicle treatment, THZ1 treatment dramatically slowed down the growth of MDA-MB-231 tumors (Fig. 7H-J). Analysis of MDA-MB231 tumors indicated that THZ1 reduced Yap level and Hippo target gene expression (Supplemental Fig. S6A,B). Expression of $\mathrm{Yki}^{-\mathrm{S} 128 \mathrm{D}}$ in MDA-MB-231 tumors resulted in higher levels of Yap protein and Hippo target gene expression compared with control tumors (Supplemental Fig. S6A,B) and rendered MDA-MB-231-YAP ${ }^{\text {S128D }}$ tumors resistant to $\mathrm{THZ1-mediated} \mathrm{inhibition} \mathrm{(Fig.} 7 \mathrm{H}-\mathrm{J})$.

\section{CDK7 inhibition reduces liver growth and tumor burden in MST1/2 DKO livers}

To further investigate the role of CDK7 in Hippo-mediated organ size control and tumor growth, we turned to mouse liver in which both MST1 and MST2 were knocked out (MST1/2 DKO). In mice, MST1/2 deficiency or Yap overexpression causes hepatocyte overproliferation, cirrhosis, and hepatocellular carcinoma (HCC) development (Dong et al. 2007; Zhou et al. 2009; Lu et al. 2010; Song et al. 2010). In humans, $50 \%$ of HCCs exhibited nuclear YAP staining, and high Yap activity correlated with poor survival after resection (Xu et al. 2009; Fitamant et al. 2015). Genetic removal of one copy of Yap or Taz restored normal growth in the Hippo pathway mutant livers (Zhang et al. 2010; Fitamant et al. 2015), suggesting that reducing Yap/Taz level was able to attenuate liver overgrowth, slow down tumor formation, and progression caused by Hpo kinase deficiency.

Treating primary hepatocytes derived from MST1/2 DKO mice with THZ1 resulted in a reduction in Yap/ Taz protein level and Hippo pathway target gene expression (Supplemental Fig. S7A), suggesting that inhibition of CDK7 could attenuate Yap/Taz activity in MST1/2 DKO hepatocytes. We next treated 1-mo-old MST1/2 DKO mice (Alb-Cre Mst1 ${ }^{-/}$Mst2 ${ }^{f l / f 1}$ ) with THZ1 $(10 \mathrm{mg} / \mathrm{kg}$ every other day) or vehicle for $2 \mathrm{mo}$. The protein level of YAP/TAZ as well as the expression of YAP/TAZ target genes SOX9, CTGF, and CYR61 in MST1/2 DKO livers were significantly down-regulated following THZ1 treatment (Fig. 7M; Supplemental Fig. S7B). In addition, the expression levels of Wnt and Notch target genes were also reduced in THZ1-treated MST1/2 DKO livers compared with control group (Supplemental Fig. S7C), consistent with a previous study showing that both Wnt and Notch signaling activities were increased in MST1/ 2 DKO livers in a Yap/Taz-dependent manner (Kim et al. 2017). Furthermore, THZ1 treatment significantly alleviated liver phenotypes caused by hepatic Yap activation, including increased organ size (Fig. 7K,L), expanded expression of oval/ductal cell markers EPCAM and Sox9 (Fig. $7 \mathrm{~N}$ ), and hepatocyte proliferation (Fig. 7O,P) compared with vehicle-treated DKO mice.

Previous studies have shown that loss of MST1/2 in hepatocytes significantly enhanced macrophages infiltration and proinflammatory cytokine expression through Yap-mediated Mcp1(Ccl2) expression, and the infiltrated macrophages promoted liver tumor initiation and progression (Guo et al. 2017; Kim et al. 2018). THZ1 treatment markedly reduced macrophage infiltration (Fig. 7Q), expression of proinflammatory cytokines (Supplemental Fig. S7D), and elevated Stat3 phosphorylation (p-Stat3) (Fig. 7M) caused by MST1/2 deficiency. Taken together, these results suggest that inhibition of CDK7 reduced liver size and tumor burden in MST1/2 DKO mice by downregulating Yap/Taz.

\section{Discussion}

The evolutionarily conserved Hippo signaling pathway controls tissue growth and organ size in diverse species and its deregulation has been implicated in a wide range of human cancer. Indeed, a recent cancer genomic study reveals that the Hippo pathway is among the eight most mutated signaling pathways in human cancer (SanchezVega et al. 2018). In addition, activation of Yap/Taz has been implicated in drug resistance in cancer treatment (Kim and Kim 2017). Hence, Yap/Taz is considered as an attractive drug target for cancer therapeutics, and understanding how Yki/Yap/Taz is regulated may provide important insight into cancer treatment. It has been well established that Yki/Yap/Taz is regulated mainly through its shuttling between the cytoplasm and the nucleus; phosphorylation by the Hippo kinase cascade sequesters Yki/Yap/Taz in the cytoplasm, whereas upstream signals leading to compromised Yki/Yap/Taz phosphorylation allows this Hippo pathway effector to enter the nucleus to activate Hippo target genes. Here we uncovered a new layer of Hippo pathway regulation consisting of a nuclear regulatory module: a Ser/Thr kinase CDK7 and an E3 ubiquitin ligase complex, CRL4 ${ }^{\text {DCAF12 }}$, which regulates Yki/Yap/Taz protein turnover and thus the duration of Hippo signaling output. We demonstrated that CDK7 phosphorylates Yki/Yap/Taz to protect them from degradation in the nucleus. In the absence of CDK7-mediated phosphorylation, Yki/Yap/Taz binds DCAF12, which recruits the E3 ubiquitin ligase complex CRL4 ${ }^{\text {DCAF12 }}$ to catalyze Yki/Yap/Taz ubiquitination, followed by proteasome-mediated degradation (Fig. 8A). An analogous mechanism has been observed in Hedgehog (Hh) signal 
A

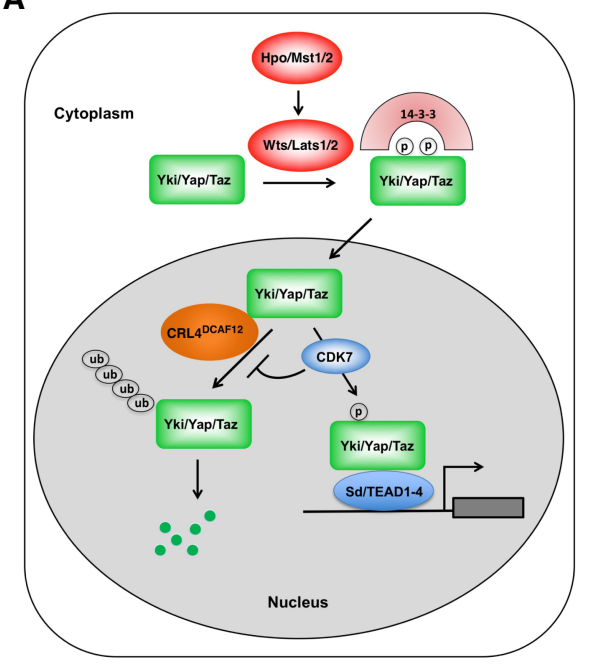

B
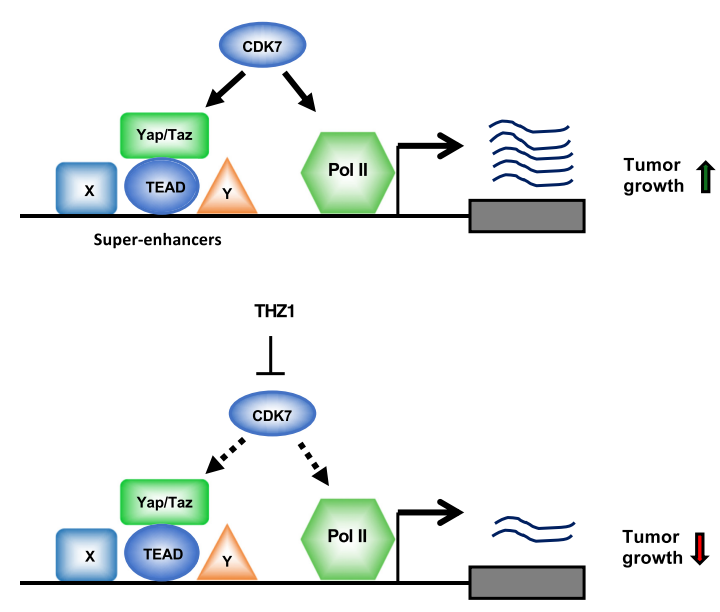

Figure 8. Targeting CDK7 for treating Yap/Taz-driven cancer. (A) A conserved role of CDK7/CRL4 ${ }^{\text {DCAF12 }}$ in the regulation of nuclear Yki/Yap/Taz. CDK7 phosphorylates Yki/Yap/Taz to prevent its binding to CRL4 ${ }^{\text {DCAF12 }}$, thereby stabilizing Yki/Yap/Taz in the nucleus. $(B, t o p)$ Yap/Taz/TEAD binds superenhancers together with other transcriptional factors $(\mathrm{X}, \mathrm{Y}$, etc.) to drive the expression of genes essential for tumor growth. (Bottom) CDK7 inhibitor THZ1 blocks tumor growth by inhibiting both Yap/Taz and Pol II in Yap/Taz-driven cancer.

transduction, in which casein kinase 1 (CK1) phosphorylates and protects the pathway transcription factor $\mathrm{Ci} /$ Gli from premature degradation by a nuclear E3 ubiquitin ligase CRL3 ${ }^{\mathrm{HIB} / \mathrm{SPOP}}$, thereby sustaining Hh pathway activity (Shi et al. 2014).

We identified CDK7 as a genetic modifier of the eye overgrowth phenotype caused by Yki overexpression. Subsequently, we found that inactivation of CDK7 in developing tissues such as wing imaginal discs resulted in reduced organ size. Several lines of evidence suggest that CDK7 regulates tissue growth and organizes size by specifically targeting the Hippo pathway effector. (1) Inactivation of CDK7 did not suppress the tissue overgrowth phenotype caused by insulin pathway activation or by overexpression of a constitutively active and Ykiindependent Sd (Sd-GA). (2) Inactivation of CDK7 down-regulated Yki protein level, leading to reduced expression of Yki target genes. (3) The reduction in wing size caused by CDK7 inactivation was reversed by restoring Yki to wild-type level. (4) Yki-driven tissue overgrowth was suppressed in a CDK7 hypomorphic mutant background in which the general transcription and cell cycle progression were not affected. Hence, even though CDK7 functions as a CAK to regulate cell cycle progression and a component of TFIIH to phosphorylate the C-terminal tail of Pol-II to influence basal transcription, we believe that under our experimental conditions, these housekeeping roles of CDK7 were preserved by residual CDK7 activity due to incomplete inactivation of CDK7. Hence, our experimental approach-partial loss of function via RNAi in a genetic sensitized background $(G M R>Y k i)$-can uncover pathway-specific role of genes (e.g., $\mathrm{CDK} 7, \mathrm{Cul} 4$, and PRP4K) with pleiotropic function (Cho et al. 2018).
Genetic epistasis experiments placed CDK7 downstream from Wts and upstream of Sd. Indeed, inactivation of CDK7 can suppress the elevated Yki activity in Wts-depleted wing discs and suppress tissue growth caused by Wts-phosphorylation deficient and constitutively active forms of Yki. Mechanistically, we demonstrated that CDK7 regulates Hippo signaling by phosphorylating Yki on S169 to protect nuclear Yki from premature loss. Using an antibody that recognizes Yki phosphorylated at S169 (pS169), we found that phosphorylation of endogenous Yki at this site was diminished but not completely abolished when CDK7 was inactivated in S2 cells or in wing imaginal discs. The residual phosphorylation at YkiS169 could be due to incomplete loss of CDK7 by RNAi or due to the presence of other kinase(s) such as Nemo-like kinase (NLK) that can also phosphorylate YkiS169 (Hong et al. 2017; Moon et al. 2017). We showed that blocking Yki phosphorylation at S169 (S169A) decreased, whereas the phosphomimetic mutation at this site (S169D) increased Yki stability and activity. Importantly, the stability and activity of these Yki variants were insensitive to either loss- or gain-of-CDK7 activity, demonstrating that $\mathrm{CDK} 7$ regulates tissue growth and organ size by modulating Hippo signaling through phosphorylating YkiS169.

Our genetic modifier screen also identified an E3 ubiquitin ligase complex (CRL4 ${ }^{\mathrm{DCAF12}}$ ) consisting of Cul4, DDB1, and DCAF12 as responsible for degrading Yki in the absence of CDK7. Strikingly, inactivation of DCAF12 suppressed the tissue growth defect caused by CDK7 inactivation by restoring Yki to wild-type level. Phosphorylation of YkiS169 by CDK7 reduced the binding of DCAF12 to and CRL4 ${ }^{\text {DCAF12 }}$-mediated ubiquitination and degradation of Yki, providing a molecular mechanism 
by which CDK7 protects nuclear Yki. We provided evidence that the CDK7/CRL4 ${ }^{\text {DCAF12 }}$ regulatory module identified in Drosophila plays a conserved role in the mammalian Hippo signaling pathway by modulating the stability and activity of Yap/Taz independent of Lats1/2. We demonstrated that CDK7-mediated phosphorylation of YapS128, and likely TazS90, increased the stability and activity of Yap/Taz by inhibiting CRL4 ${ }^{\text {DCAF12 }}$-mediated ubiquitination of Yap/Taz. Pharmacological inhibition of CDK7 in MST1/2 DTO liver decreased Yap/Taz protein level and transcriptional activity and reversed the overproliferation phenotype caused by Hippo signaling deficiency. Hence, CDK7-dependent Yap/Taz stabilization many represent an Achilles heel in the Hippo signaling pathway that can be explored therapeutically. It remains to be determined whether Yap/Taz phosphorylation by CDK7 is a regulated event under physiological and/or pathological conditions. Interestingly, recent studies reported that CDK7 is up-regulated in cancer and is associated with poor prognosis (Li et al. 2017; Jiang et al. 2019). On the other hand, many cancer cells have high levels of nuclear Yap/Taz without harboring identifiable Hippo pathway mutations (Zanconato et al. 2016a). Therefore, it would be interesting to determine whether up-regulation of CDK7 or down-regulation of CRL4 ${ }^{\text {DCAF12 }}$ could contribute to elevated nuclear Yap/Taz in cancer cells.

Hippo pathway mutations and Yap amplification that lead to increased Yap/Taz activity have been attributed to many types of human cancer, placing Yap/Taz as a prominent anticancer drug target; however, transcription factors and cofactors that do not possess enzymatic activity have been proved difficult to target and belong to the so called "nondruggable class." Our finding that a Ser/Thr kinase CDK7 is required for Yap/Taz stabilization and activity in the nucleus raises an exciting possibility that targeting this Hippo pathway vulnerability could represent a new therapeutic strategy to combat cancers caused by Hippo pathway deregulation. Indeed, we found that depletion of CDK7 in several Yap/Taz-driven cancer cell lines including MDA-MB-231, HCT116, and EC9706 inhibited cancer cell proliferation and invasiveness. Furthermore, we found that pharmacological inhibition of CDK7 by a small molecule THZ1 blocked MDA-MB-231 tumor growth in Xenografts, and that this inhibitory effect was largely reversed by expressing a phospho-mimetic and

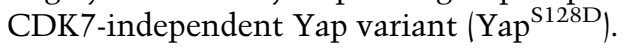

Several recent studies demonstrated that THZ1 and its derivative THZ2 exhibited selective inhibitory effect on tumor growth in a number of preclinic cancer models including T-cell acute lymphoblastic leukemia (T-ALL) (Kwiatkowski et al. 2014), MYCN-driven neuroblastoma (Chipumuro et al. 2014), small cell lung cancer (SCLC) (Christensen et al. 2014), TNBC (Wang et al. 2015; Li et al. 2017), esophageal squamous cell carcinoma (Jiang et al. 2017), epithelial ovarian cancer (EOC) (Francavilla et al. 2017), diffuse intrinsic pontine glioma (DIPG) (Nagaraja et al. 2017), and HCC (Zhong et al. 2018; Tsang et al. 2019). While many cancer cell lines are sensitive to THZ1, others such as estrogen receptor positive breast cancer cells remained resistant, although the underlying mechanisms remain unknown (Kwiatkowski et al. 2014; Wang et al. 2015). It is thought that CDK7 inhibition affects cancer progression by interfering with the expression of genes associated with superenhancers, which are sensitive to reduced Pol-II CTD phosphorylation (Chipumuro et al. 2014; Wang et al. 2015). However, a previous study indicated that THZ1 could inhibit cancer cell growth at low doses that did not significantly affect Pol-II CTD phosphorylation (Kwiatkowski et al. 2014). In addition, CDK7 KO mice did not affect significantly Pol-II CTD phosphorylation likely due to the compensation by other kinases (Ganuza et al. 2012). Hence, CDK7 inhibition may affect superenhancer-associated gene expression through mechanisms other than or in addition to inhibiting PolII CTD phosphorylation. Interestingly, a recent study revealed that Yap/Taz occupied a large set of enhancers with superenhancer-like property in TNBC cells to mediate cancer transcriptional addiction (Zanconato et al. 2018). Our finding that inactivation of CDK7 destabilizes Yap/Taz provides an additional mechanism and alternative explanation of why CDK7 inhibition tends to affect superenhancer-driven gene expression and suggests that Yap/Taz-driven cancers have become more "addicted" to CDK7 activity (Fig. 8B). Our finding also suggests that partial inhibition of CDK7 could preferentially affect Yap/Taz target genes and thus Yap/Taz-driven cancer growth without perturbing the general transcription and cell cycle progression in normal cells as we have demonstrated in Drosophila. In addition, we suggest that high Yap/Taz expression may serve as a biomarker for cancers sensitive to CDK7 inhibitors. It would be interesting to determine whether CDK7 could phosphorylate additional transcriptional factors/cofactors bound to superenhancers to influence gene expression in cancer.

Our finding that CRL4 ${ }^{\text {DCAF12 }}$ bind unphosphorylated Yap/Taz to target it for ubiquitination and degradation also has an important therapeutic implication. Small molecules that can promote the binding of the CRL4 family of E3s to oncogenic substrates have been explored as anticancer drugs (Fischer et al. 2014; Han et al. 2017). Therefore, searching for small molecules that can increase the binding of CRL4 ${ }^{\text {DCAF12 }}$ to Yap/Taz, especially the phosphorylated form of Yap/Taz may provide a new strategy to identify anticancer drugs. On the other hand, inhibition of CRL4 ${ }^{\text {DCAF12 }}$-mediated degradation of Yap/Taz may enhance Yap/Taz activity in the nucleus, which could be beneficial for tissue regeneration.

\section{Materials and methods}

Drosophila genetics and transgenes

Fly culture and crosses were carried out according to standard procedures. The transgenic RNAi lines used were UAS-CDK7RNAi (VDRC\#10442, VDRC\#103413, BL\#62304); UAS-CycHRNAi (VDRC\#104312, BL\#34732); UAS-Mat1-RNAi (VDRC\# 104780, BL\#57312); UAS-Xpd1-RNAi (VDRC\#106998, BL\#658 83); UAS-Cul4-RNAi (v105668, v44829); UAS-DCAF12-RNAi (VDRC\#43758); UAS-Wts-RNAi (VDRC\#106174). The 
transgenes used were UAS-Yki ${ }^{W T}, U A S-Y k i^{S 168 A}$, and UAS-SdGA (Zhang et al. 2008); UAS-Yki ${ }^{S 111 A S 250 A}$ and UAS-Yki ${ }^{3 S A}$ (Ren et al. 2010); UAS-InR ${ }^{A C}$ (BL\#8263). UAS-GFP-CDK7 and $U A S-G F P-C D K 7^{D R}$ were generated by P-element mediated transformation. $U A S-Y k i^{W T}, U A S-Y k i^{S 169 A}$ and $U A S-Y k i^{S 169 D}$ were inserted at the 75B1attP locus (Bischof et al. 2007). The Hippo pathway reporters used were diap-GFP3.5, 3xSd2-Luc (Zhang et al. 2008); ex-lacZ (Hamaratoglu et al. 2006); diap1-lacZ $\left(t h^{i 5 c 8}\right)$ (Ryoo et al. 2002); Yki:GFP (Fletcher et al. 2018). The Gal4 drivers used were GMR-Gal4 (Freeman 1996), and hh-Gal4 (Zhang et al. 2008), MS1096 (Wang et al. 1999). The CDK7 ${ }^{\text {S164A/ }}$ ${ }_{T 170 A}$ mutant flies $W D f(1) J B 254 / W D f(1) J B 254$ (deficiency for $C D K 7) ; \quad P W^{+}\left(C D K 7^{S 164 A / T 170 A}\right) / P_{W^{+}}(c d k 7$ S164A/T170A) were obtained from Dr. Hyung Don Ryoo (Morishita et al. 2013). Because CDK7 is on the X chromosome, $C D K 7^{S 164 A / T 170 A}$ mutant females or control flies were crossed to $G M R>Y k i$ males and their male progenies were analyzed.

\section{DNA constructs}

The $p U A S T$ constructs used were UAS-Myc-Yki (Zhang et al. 2008); UAS-Fg-Cul4, and UAS-Fg-DDB1 (Li et al. 2018). MyrYki-GFP contained a myristoylation signal MGNKCCSKRQ and GFP tag at the $\mathrm{N}$ and $\mathrm{C}$ terminus, respectively. Myr-MycYki contained a myristoylation signal at the $\mathrm{N}$ terminus of Myc-Yki. YkiS169A and YkiS169D were generated by PCR-mediated site-directed mutagenesis. Drosophila CDK7 cDNA clone was obtained from DGRC (Flybase). CDK $7{ }^{\mathrm{DR}}$ (D137R) variant was generated by PCR-mediated site-directed mutagenesis. GFP or HA tag was inserted at the $\mathrm{C}$ terminus of $\mathrm{CDK} 7$ or $\mathrm{CDK} 7{ }^{\mathrm{DR}}$ to generate GFP/HA-CDK7 or GFP/HA-CDK $7{ }^{\mathrm{DR}}$. The constructs were subcloned into the $p U A S T$ vector digested with NotI and XbaI. pcDNA3.1 construct expressing Fg-Yap or HA-Taz (Cho et al. 2018). pcDNA3.1 construct expressing human HA-CDK7 (Addgen). To generate HA-DCAF12 construct, DNA fragments encoding HA-DCAF12 was amplified by PCR and inserted between KpnI and EcoRI sites of the pCDNA3.1 vector. FgYapS128A, Fg-YapS128D, HA-TazS90A, and HA-TazS90D were generated by PCR-mediated site-directed mutagenesis. The constructs were subcloned into $p c D N A 3.1$ for transient transfection experiments. To make GST-Yki fusion constructs, synthetic DNA oligos corresponding to the coding sequences for aal60181 and their SA mutants (S168A, S169A, and S172A) were annealed and subcloned into $p G E X 4 T-1$ vector digested with EcoRI and XhoI.

\section{Cell culture, transfection, and lentiviral production}

$\mathrm{S} 2\left(\mathrm{~S} \mathrm{R}^{+}\right)$cells were cultured in Schneider's Drosophila medium (Life Technologies) with 10\% fetal bovine serum (GE Healthcare), $100 \mathrm{U} / \mathrm{mL}$ penicillin (Life Technologies), and $100 \mathrm{mg} / \mathrm{mL}$ streptomycin (Life Technologies) at $24^{\circ} \mathrm{C}$. Transfection of S2 cells was performed using calcium phosphate transfection kit (Specialty Media) following the manufacturer's instruction. A ubiquitinGal4 construct was cotransfected with $p U A S T$ constructs to drive UAS transgene expression in S2 cells. HEK293A, MDAMB-231, EC9706, and HC116 cells were obtained from the American Type Culture Collection (ATCC). These cancer cells were cultured in Dulbecco's modified Eagle's medium (DMEM) (Life Technologies, 41965) supplemented with $10 \%$ fetal bovine serum (FBS, 10270, Life Technologies) and 1\% penicillin/streptomycin. All cell lines were subject to cell line authentication via short tandem repeat (STR), which was performed via PowerPlex21 system and compared with STR data in ATCC. Lats1/2 ${ }^{-/-}$HEK293 cells were obtained from Dr. Kun-Liang Guan (Meng et al. 2015).
HEK293 cells were cultured in DMEM (Sigma-Aldrich) containing $10 \%$ BCS (ATCC), and transfected using GenJet Plus in vitro DNA transfection kit (SignaGen). For starvation experiments, HEK293A cells were cultured in DMEM (Sigma-Aldrich) without serum for $6 \mathrm{~h} 2 \mathrm{~d}$ after transfection. For lentivirus infection, $\mathrm{Yap}^{\mathrm{WT}}$ and Yap ${ }^{\mathrm{S} 128 \mathrm{D}}$ were cloned into $p L V X-I R E S-Z$ Sgreen vector. The expression vectors and package vectors (PSPAX2 and PMDG2) were cotransfected into HEK293T cells with lipofectamine 2000 (Thermo Fisher Scientific, cat: 11668). After 48 h, the recombinant viruses were harvested for cell line infection. The recombinant viruses were infected into MDA-MB-231 cells using the standard method.

\section{RNA interference}

For RNAi experiments in S2 cells, dsRNAs were generated using the MEGAscript high-yield transcription kit (Ambion). The following primers were used for generating the dsRNA targeting individual genes: CDK7 (5'-GAATTAATACGACTCACTATAG GGAGATGAACTTATGCTGCGAGTGC-3' and 5'-GAATTAA TACGACTCACTATAGGGAGAATTGCCCCTCACCGTAGT CT- $\left.3^{\prime}\right)$ and DCAF12 (5'-GAATTAATACGACTCACTATAGG GAGACGCGAATTCCGCCATCCCAACGCTCTCAA-3' and 5'-GAATTAATACGACTCACTATAGGGAGAGCGTCTAGAC TCGCCGCCATCTGGAAACTC-3'). dsRNA targeting the coding sequence of luciferase was used as a control. For RNAi in mammalian cells, siRNA was transfected into cells using Lipofectamine RNAi-MAX (cat:13778, Invitrogen) in antibiotics-free medium according to manufacturer's instruction. Oligonucleotides of siRNA duplexes were purchased from Sigma as follows: siCDK7\#1 (SASI_Hs_01_00214780), siCDK7\#2 (SASI_Hs_01_ 00214781), siDCAF12 \#1 (SASI_Hs01_00216314), siDCAF12 \#2 (SASI_Hs01_00216315), siDCAF12 \#3 (SASI_Hs01_00216316), and siDCAF12 \#4 (SASI_Hs01_00216317). The siControl used was UUCUCCGAACGUGUCACGUTT.

\section{Immunoprecipitation and Western blotting}

For immunoprecipitation assay, cells were harvested and washed twice with PBS after transfection for $48 \mathrm{~h}$ and then lysed on ice for 30 min with lysis buffer containing $1 \mathrm{M}$ Tris $\mathrm{pH} 8.0,5 \mathrm{M} \mathrm{NaCl}, 1 \mathrm{M}$ $\mathrm{NaF}, 0.1 \mathrm{M} \mathrm{Na}_{3} \mathrm{VO}_{4}, 1 \% \mathrm{NP}-40,10 \%$ glycerol, and 0.5M EDTA (pH 8.0). Cell lysates were incubated with protein A-Sepharose beads (Thermo scientific) for $1 \mathrm{~h}$ at $4^{\circ} \mathrm{C}$ to eliminate nonspecific binding proteins. After removal of the protein-A beads by centrifugation, the cleared lysates were incubated with Myc (HA or Flag) antibody for $2 \mathrm{~h}$ or overnight. The complexes were collected by incubation with protein A-Sepharose beads for $1 \mathrm{~h}$ at $4^{\circ} \mathrm{C}$, followed by centrifugation. The immunoprecipitates were then washed three times for 5 min each with lysis buffer and were separated on SDS-PAGE. Western blot was carried out using standard protocol. The cancer cells were lysed with RIPA buffer. Lysis samples were separated by electrophoresis on SDS-PAGE and electrotransferred to PVDF membrane. Mouse liver tissues or tumors were lysed in RIPA buffer containing protease inhibitor cocktail (Roche). Lysates were clarified by centrifugation at $18,500 \mathrm{~g}$ for 10 min at $4^{\circ} \mathrm{C}$, and protein concentration was determined by Pierce BCA protein assay kit (Thermo Fisher Scientific). The boiled samples were separated on Bis-Tris protein gels and transferred to nitrocellulose membranes (Life Technologies, Thermo Fisher Scientific). After blocking with $5 \%$ BSA in TBS with $0.5 \%$ Tween- 20 for $1 \mathrm{~h}$, the membranes were probed with the corresponding antibodies overnight. Bound antibodies were visualized by ECL (EMD Millipore or Pierce, Thermo Fisher Scientific) using HRP-conjugated antibodies. 
Immunofluorescence

Immunostaining of imaginal discs was carried out as previously described (Jiang and Struhl 1995). For immunostaining of cultured cells, cells were seeded on LAB-TEK chamber slides, transfected with indicated constructs, and stained using standard protocols. Liver tissues were perfused with ice-cold PBS and fixed in $4 \%$ paraformaldehyde for the preparation of cryosections. Sections were permeabilized in $0.5 \%$ Triton/PBS (PBST) for $5 \mathrm{~min}$, followed by three washes in PBS. Sections were incubated in blocking buffer (1\% BSA in PBST) for $1 \mathrm{~h}$ at room temperature and then incubated overnight at $4^{\circ} \mathrm{C}$ with the indicated primary antibodies. Sections were washed five times in PBS and then incubated with a secondary antibody for $1 \mathrm{~h}$ at room temperature. Donkey antimouse Alexa fluor 488, donkey antirat Alexa fluor 594, or donkey antirabbit Alexa fluor 594 secondary antibodies (Life Technologies, Thermo Fisher Scientific) were used. Sections were washed 10 times in PBST and then mounted with mounting medium with DAPI (catalog H-1200; Vector Laboratories). Immunofluorescence imaging was performed using a Zeiss 510 NLO META laser-scanning microscope.

\section{Antibodies}

Yki S169 phospho-specific antibody (pS169) was generated using IHHSRARS(pS)PASLQQN as antigen and affinity purified by Gememed Synthesis. Other antibodies used were rabbit antiYki (Li et al. 2015), Yap S128 phospho-specific antibody (pS128) (Moon et al. 2017), mouse anti- $\beta$-Gal (Promega), rabbit anti- $\beta$ Gal (Affinity Bioreagents), mouse anti-Wg (Developmental Studies Hybridoma Bank [DSHB]), rat anti-Ci, 2A1 (DSHB), rabbit antiGFP (Invitrogen), mouse anti-Myc (Santa Cruz Biotechnology), rabbit and mouse anti-Flag (Sigma), mouse anti-HA (Santa Cruz Biotechnology), rabbit anti-Yap (Abcam). rabbit anti-CDK7 (Cell Signaling), mouse anti-Yap/TAZ (Santa Cruz Biotechnology, catalog sc-10119), mouse anti-TAZ (Santa Cruz Biotechnology, catalog sc-518026), mouse anti-Actin(Cell Signaling, catalog 3700), rabbit anti-Yap/Taz (Cell Signaling, catalog 8418), rabbit anti-pYap (Cell Signaling, catalog 4911), rabbit anti-p-STAT3 (Cell Signaling, catalog 9145), mouse anti-GAPDH (Sigma-Aldrich, catalog SAB1405848), rabbit anti-p-Histone3 (Cell Signaling, catalog 9701), goat anti-CTGF (Santa Cruz Biotechnology, sc-14940), goat anti-CYR61(Santa Cruz Biotechnology, sc-8561), rabbit anti-SOX9 (EMD Millipore, catalog AB5535), rabbit anti-Ki67 (Invitrogen, catalog PA5-16785), rat anti-EPCAM (DSHB, clone G8.8), and rat anti-F4/80 (AbD Serotec, catalog MCA497). Antimouse and rabbit HRP-conjugated secondary antibodies were from GE Healthcare Life Sciences. Antigoat and antirat HRP-conjugated secondary antibodies were from Santa Cruz Biotechnology, Inc., and Sigma-Aldrich, respectively.

Mouse lines, xenografts, and drug treatment.

The procedures for all animal experiments were reviewed and approved by the Institutional Animal Care and Use Committees of Harvard Medical School and University of Texas Southwestern Medical School. The Alb-Cre Mst1 ${ }^{-/-} M s t 2^{f 1 / f 1}$ mouse lines have been described previously (Dong et al. 2007; Zhou et al. 2009; $\mathrm{Lu}$ et al. 2010; Song et al. 2010). Both male and female mice were used, which did not show difference in this study. Mice were treated with THZ1 (APEXBIO, catalog no. A8882) by intraperitoneal injection at a concentration of $10 \mathrm{mg} / \mathrm{kg}$ every other day or in equivalent volumes of vehicle as a control. Mice received 28 injections during the 8 -wk period starting at 1 mo of age. For TNBC xenograft experiments, MDA-MB-231 cells infected with control vector and Yap ${ }^{\text {S128D }}$ lentivirus were FACS sorted, and $1 \times 10^{6}$ of each was suspended in $100 \mu \mathrm{L}$ of PBS with $40 \%$ Matrigel (BD Biosciences) and injected into the fourth pair of mammary fat pads of 6-wk-old female NSG mice (purchased from the University of Texas Southwestern Medical Center animal breeding core facility). Once the tumor size reached $\sim 100$ $\mathrm{mm}^{3}$, the animals for each cell line were randomly divided into two groups (seven mice/ group) and treated with either vehicle (10\% DMSO in D5W, $5 \%$ dextrose in water) or $10 \mathrm{mg} / \mathrm{kg}$ THZ1 (MedChemExpress, catalog no. HY-80013) through intraperitoneal administration twice daily. Growth in tumor volume was measured with digital calipers every $3 \mathrm{~d}$. Tumor volumes were estimated using the formula $\mathrm{V}=$ length $\times$ width $2 \times 0.5$. At the end of the studies, mice were sacrificed for tumor collection, tumors were weighed and frozen in liquid nitrogen for future analysis.

\section{Hepatocyte isolation and treatment}

Primary hepatocytes were isolated from Alb-Cre Mst1 ${ }^{-/-}$Mst $2^{f 1 / f 1}$ (DKO) mouse via collagenase-elastase perfusion according to the manufacturer's protocol (Worthington Biochemical). Briefly, mice were anesthetized with sodium pentobarbital solution (30 $\mathrm{mg} / \mathrm{kg}$ i.p.) and their livers perfused with calcium- and magnesium-free HBSS (CMF-HBSS), followed by enzyme buffer solution ( $225 \mathrm{U} / \mathrm{mL}$ collagenase, $0.3 \mathrm{U} / \mathrm{mL}$ elastase, and $10 \mathrm{U} / \mathrm{mL}$ DNase). The isolated mouse hepatocytes were then cultured in DMEM containing $10 \%$ FBS, l-glutamin, and penicillin-streptomycin in collagen-coated plates (Biocoat; BD). Primary hepatocytes were treated with THZ1 $(500 \mathrm{nM})$ for $12 \mathrm{~h}$, and cell lysates were subjected to Western blot analysis using indicated antibodies.

\section{$R T-q P C R$}

Total RNA was extracted using RNeasy Plus mini kit (Qiagen \#74134), and cDNA was synthesized using the iScript cDNA synthesis kit (Bio-Rad). RT-qPCR was performed using iQ SYBR Green system (Bio-Rad) and a Bio-Rad CFX96 real-time PCR system.

36B4 expression level was used a normalization control. Primer sequences used were CTGF (CATCTTCGGTGGTACGGTGT $[\mathrm{F}]$ and TTCCAGTCGGTAAGCCGC [R]), CYR61 (CGGGTTT CTTTCACAAGGCG [F] and TGAAGCGGCTCCCTGTTTTT [R]), ANKRD1 (GCCATGCCTTCAAAATGCCA [F] and AGAA CTGTGCTGGGAAGACG [R]), AJUBA (TACCAGGACGAGC TAACAGC $[\mathrm{F}]$ and TACAGGTGCCGAAGTAGTCC $[\mathrm{R}])$, and 36B4 (GGCGACCTGGAAGTCCAACT [F] and CCATCAGCAC CACAGCCTTC $[\mathrm{R}])$.

Total RNA from mouse liver tissue was prepared using RNAzol reagent (RNAzol RT, MRC) according to the manufacturer's protocol. cDNA was synthesized from total RNA (1-2 $\mu \mathrm{g})$ using highcapacity cDNA reverse transcription kit (Life Technologies, Thermo Fisher Scientific). qRT-PCR was performed using SYBR Select Master Mix (Thermo Fisher Scientific) on a StepOnePlus thermal cycler from Applied Biosystems. Expression levels were always given relative to GAPDH. The following PCR primers for mouse samples were used: Ctgf (forward, CTGCCTACC GACTGGAAGAC; reverse, CATTGGTAACTCGGGTGGAG), Cyr61 (forward, GCTCAGTCAGAAGGCAGACC; reverse, GT TCTTGGGGACACAGAGGA), Axin2 (forward, GCTGGAGAA ACTGAAACTGGA; reverse, CAAAGTGTTGGGTGGGGTA AG), Wif1 (forward, GCCACGAACCCAACAAGT; reverse, TCCСTTCTATCCTCAGCCTTT), Hes1 (forward, GTGGGTC CTAACGCAGTGTC; reverse, TCAGAAGAGAGAGGTGGG CTA), Il6 (forward, TCCATCCAGTTGCCTTCTTG; reverse, TTCCACGATTTCCCAGAGAA), Lif (forward, ATTGTGCC 
CTTACTGCTGCTG; reverse, GCCAGTTGATTCTTGATCT GGT), I11b (forward, CAACCAACACGTGATATTCTCCATG; reverse, GATCCACACTCTCCAGCTGCA), EpCam (forward, CCTGAGAGTGAACGGAGAGC; reverse, GACACCACCAC AATGACAGC), Sox9 (forward, CGACTACGCTGACCATCA GA; reverse, AGACTGGTTGTTCCCAGTGC), Gapdh (forward, ATCCTGCACCACCAACTGCT; reverse, GGGCCATCCACA GTCTTCTG), Tgf- $\beta 1$ (forward, GCCTGAGTGGCTGTCTTT TGA; reverse, GGCTGATCCCGTTGATTTCCA), Tgf- $\beta 2$ (forward, TGGAGTTCAGACACTCAACACA; reverse, AAGCTT CGG GATTTATGGTGT), Tgf- $\beta 3$ (forward, AGTGGCTGTTGA GGAGAGAGT; reverse, GTGTGGGTTGTGGTG ATCCTT), F4/80 (forward, CCCCAGTGTCCTTACAGAGTG; reverse, GTGCCCAGAGTGGATGTCT), c- Myc (forward, ATGCCCC TCAACGTGAACTTC; reverse, CGCAACATAGGATGGAGA GCA), Acta2 (forward, ATGCTCCCAGGGCTGTTTTCCCAT; reverse, GTGGTGCCAGATCTTTTCCATGTCG), and Gapdh (forward, ATCCTGCACCACCAACTGCT; reverse, GGGCC ATCCACAGTCTTCTG.

\section{In vitro kinase assay}

In vitro kinase assay was carried out by incubating $25 \mu \mathrm{L}$ of reaction mixtures containing $150 \mathrm{mM}$ Tris- $\mathrm{HCl}(\mathrm{pH} 7.5), 0.2 \mathrm{mM} \mathrm{Mg}^{2}$ +/ATP, $1 \mu \mathrm{g}$ of purified GST-Yki fusion proteins, together with appropriate amount of GFP-CDK7 or GFP-CDK $7^{\mathrm{DR}}$ immunopurified $\mathrm{S} 2$ cells for $1.5 \mathrm{~h}$ at $30^{\circ} \mathrm{C}$. The reaction was terminated by adding $2 \times$ SDS loading buffer. The resultant samples were load on SDS-PAGE and subjected to pIMAGO phospho-protein detection kit with fluor-680 (Sigma)

\section{Luciferase reporter assay}

To measure the activity of Yki/YAP/TAZ, cells were seeded in 24-well plates and co-transfected with a $S d$ (Scalloped)-dependent luciferase reporter construct, 3XSd2-luc (Zhang et al. 2008), with pTK-Renilla and effector plasmids. The luciferase activities were analyzed using a dual-luciferase reporter assay kit (Promega) according to manufacturer's instruction.

\section{Ubiquitination assay}

For Yki ubiquitination assay, S2 cells were treated with $50 \mu \mathrm{M}$ MG132 (Calbiochem) for $4 \mathrm{~h}$ prior to harvest. Cells were lysed in lysis buffer (150 mM Tris pH 8.0, $100 \mathrm{mM} \mathrm{NaCl}, 10 \mathrm{mM}$ $\mathrm{NaF}, 1 \mathrm{mM} \mathrm{Na} 3 \mathrm{VO} 4,10 \%$ Glycerol, $1.5 \mathrm{mM}$ EDTA/1) for 30 min on ice. After centrifugation, SDS was added to the supernatants at $1 \%$ final concentration. After boiling for $3 \mathrm{~min}$, the lysates were diluted 10-fold with lysis buffer and then subjected to immunoprecipitation and Western blot analysis. For Yap/Taz ubiquitination assay, HEK293T cells were lysed with lysis buffer containing $50 \mathrm{mM}$ Tris $\mathrm{HCl}(\mathrm{pH} 8), 100 \mathrm{mM} \mathrm{NaCl}, 10 \mathrm{mM} \mathrm{NaF}, 1$ mM Na3VO4, 1\% IGEPAL CA-630, 10\% glycerol, 1.5 mM EDTA (1 mL for one $10-\mathrm{cm}$ plate). After centrifugation to remove cell debris and nuclei, $225 \mu \mathrm{L}$ of Supernatant was mixed with $25 \mu \mathrm{L}$ $10 \%$ SDS and boiled for $3 \mathrm{~min}$. One milliliter of the above lysis buffer was added prior to immunoprecipitation and Western blot analysis.

\section{Cell proliferation assay}

MDA-MB-231, EC9706, and HCT116 cells were seeded and transfected with $50 \mu \mathrm{M}$ CDK7 siRNA or control siRNA. Twenty-four hours after transfection, cells were counted to ensure that the same number of cells were seeded into 96-well plates. Cell num- bers were determined using WST-1 cell proliferation reagent (Sigma-Aldrich, cat: 5015944001) at indicated time points.

Transwell invasion assay

Two-chamber plates (\#3422, Corning) were used to perform the transwell assay. For the transwell assay, cells were transfected with $50 \mathrm{nmol} / \mathrm{L}$ siRNA or siControl. After $24 \mathrm{~h}, 1 \times 10^{5}$ control cells and CDK7 knocking-down cells were put into the chamber. To stimulate the invasion, complete medium was added to the bottom wells, while no FBS medium was added in the upper chamber. After $24 \mathrm{~h}$ of incubation at $37^{\circ} \mathrm{C}$ for invasion assay, cells in the upper chamber were carefully removed and the cells that had shuttled into the membrane were fixed and stained with Crystal Violet staining solution. Cellular quantification was analyzed in three fields with $100 \times$ magnification under a microscope. The quantification was based on cell-number counting in each vision.

\section{Acknowledgments}

We thank Dr. H.D. Ryoo, Dr. E.H. Jho, Dr. K.L. Guan, and Dr. B. Thompson for reagents; Bloomington and VDRC stock centers for fly stocks; and DSHB for antibodies. This work was supported by grants from the National Institutes of Health (GM118063) and the Welch Foundation (I-1603) to J.J. and the National Cancer Institute (CA222571) to Y.Y. J.J. is a Eugene McDermott Endowed Scholar in Biomedical Science at the University of Texas Southwestern.

Author Contributions: Y.C. and J.J. conceived the study. Y.C., S.L., J.Z., Y.Y., and J.J. were responsible for the methodology. Y.C., S.L., X.W., J.Z., S.Z., Y.H., and T.Y. performed the investigations. Y.C., S.L., J.Z., Y.Y., and J.J. acquired the resources. J.J. wrote the manuscript. Y.C., S.L., X.W., J.Z., S.Z., and Y.H. visualized the study. Y.Y. and J.J. acquired the funding. Y.Y. and J.J. supervised the study.

\section{References}

Angers S, Li T, Yi X, MacCoss MJ, Moon RT, Zheng N. 2006. Molecular architecture and assembly of the DDB1-CUL4A ubiquitin ligase machinery. Nature 443: 590-593. doi:10.1038/ nature 05175

Bischof J, Maeda RK, Hediger M, Karch F, Basler K. 2007. An optimized transgenesis system for Drosophila using germ-linespecific $\varphi \mathrm{C} 31$ integrases. Proc Natl Acad Sci 104: 33123317. doi:10.1073/pnas.0611511104

Chang SS, Yamaguchi H, Xia W, Lim SO, Khotskaya Y, Wu Y, Chang WC, Liu Q, Hung MC. 2017. Aurora A kinase activates YAP signaling in triple-negative breast cancer. Oncogene 36: 1265-1275. doi:10.1038/onc.2016.292

Chang L, Azzolin L, Di Biagio D, Zanconato F, Battilana G, Lucon Xiccato R, Aragona M, Giulitti S, Panciera T, Gandin A, et al. 2018. The SWI/SNF complex is a mechanoregulated inhibitor of YAP and TAZ. Nature 563: 265-269. doi:10.1038/s41586018-0658-1

Chipumuro E, Marco E, Christensen CL, Kwiatkowski N, Zhang T, Hatheway CM, Abraham BJ, Sharma B, Yeung C, Altabef A, et al. 2014. CDK7 inhibition suppresses super-enhancerlinked oncogenic transcription in MYCN-driven cancer. Cell 159: 1126-1139. doi:10.1016/j.cell.2014.10.024

Cho YS, Zhu J, Li S, Wang B, Han Y, Jiang J. 2018. Regulation of Yki/Yap subcellular localization and Hpo signaling by a 
nuclear kinase PRP4K. Nat Commun 9: 1657. doi:10.1038/ s41467-018-04090-2

Christensen CL, Kwiatkowski N, Abraham BJ, Carretero J, AlShahrour F, Zhang T, Chipumuro E, Herter-Sprie GS, Akbay EA, Altabef A, et al. 2014. Targeting transcriptional addictions in small cell lung cancer with a covalent CDK7 inhibitor. Cancer Cell 26: 909-922. doi:10.1016/j.ccell.2014.10.019

Dong J, Feldmann G, Huang J, Wu S, Zhang N, Comerford SA, Gayyed MF, Anders RA, Maitra A, Pan D. 2007. Elucidation of a universal size-control mechanism in Drosophila and mammals. Cell 130: 1120-1133. doi:10.1016/j.cell.2007.07 .019

Fang L, Teng H, Wang Y, Liao G, Weng L, Li Y, Wang X, Jin J, Jiao C, Chen L, et al. 2018. SET1A-mediated mono-methylation at K342 regulates YAP activation by blocking its nuclear export and promotes tumorigenesis. Cancer Cell 34: 103-118.e9. doi:10.1016/j.ccell.2018.06.002

Fischer ES, Böhm K, Lydeard JR, Yang H, Stadler MB, Cavadini S, Nagel J, Serluca F, Acker V, Lingaraju GM, et al. 2014. Structure of the DDB1-CRBN E3 ubiquitin ligase in complex with thalidomide. Nature 512: 49-53. doi:10.1038/nature 13527

Fisher RP. 2005. Secrets of a double agent: CDK7 in cell-cycle control and transcription. J Cell Sci 118: 5171-5180. doi:10 $.1242 /$ jcs. 02718

Fitamant J, Kottakis F, Benhamouche S, Tian HS, Chuvin N, Parachoniak CA, Nagle JM, Perera RM, Lapouge M, Deshpande V, et al. 2015. YAP inhibition restores hepatocyte differentiation in advanced HCC, leading to tumor regression. Cell Rep 10: 1692-1707. doi:10.1016/j.celrep.2015.02.027

Fletcher GC, Diaz-de-la-Loza MD, Borreguero-Muñoz N, Holder M, Aguilar-Aragon M, Thompson BJ. 2018. Mechanical strain regulates the Hippo pathway in Drosophila. Development 145: dev159467. doi:10.1242/dev.159467

Francavilla C, Lupia M, Tsafou K, Villa A, Kowalczyk K, Rakownikow Jersie-Christensen R, Bertalot G, Confalonieri S, Brunak S, Jensen LJ, et al. 2017. Phosphoproteomics of primary cells reveals druggable kinase signatures in ovarian cancer. Cell Rep 18: 3242-3256. doi:10.1016/j.celrep.2017.03.015

Freeman M. 1996. Reiterative use of the EGF receptor triggers differentiation of all cell types in the Drosophila eye. Cell 87: 651-660. doi:10.1016/S0092-8674|00|81385-9

Ganuza M, Sáiz-Ladera C, Cañamero M, Gómez G, Schneider R, Blasco MA, Pisano D, Paramio JM, Santamaría D, Barbacid M. 2012. Genetic inactivation of Cdk7 leads to cell cycle arrest and induces premature aging due to adult stem cell exhaustion. EMBO J 31: 2498-2510. doi:10.1038/emboj.2012.94

Guo X, Zhao Y, Yan H, Yang Y, Shen S, Dai X, Ji X, Ji F, Gong XG, Li L, et al. 2017. Single tumor-initiating cells evade immune clearance by recruiting type II macrophages. Genes Dev 31: 247-259. doi:10.1101/gad.294348.116

Halder G, Johnson RL. 2011. Hippo signaling: growth control and beyond. Development 138: 9-22. doi:10.1242/dev .045500

Halder G, Callaerts P, Flister S, Walldorf U, Kloter U, Gehring WJ. 1998. Eyeless initiates the expression of both sine oculis and eyes absent during Drosophila compound eye development. Development 125: 2181-2191.

Hamaratoglu F, Willecke M, Kango-Singh M, Nolo R, Hyun E, Tao C, Jafar-Nejad H, Halder G. 2006. The tumour-suppressor genes NF2/Merlin and expanded act through Hippo signalling to regulate cell proliferation and apoptosis. Nat Cell Biol 8: 27-36. doi:10.1038/ncb1339

Han T, Goralski M, Gaskill N, Capota E, Kim J, Ting TC, Xie Y, Williams NS, Nijhawan D. 2017. Anticancer sulfonamides target splicing by inducing RBM39 degradation via recruitment to DCAF15. Science 356: eaal3755.

Harvey KF, Pfleger CM, Hariharan IK. 2003. The Drosophila Mst ortholog, hippo, restricts growth and cell proliferation and promotes apoptosis. Cell 114: 457-467. doi:10.1016/S0092-8674 (03)00557-9

Hong AW, Meng Z, Yuan HX, Plouffe SW, Moon S, Kim W, Jho EH, Guan KL. 2017. Osmotic stress-induced phosphorylation by NLK at Ser128 activates YAP. EMBO Rep 18: 72-86. doi:10 $.15252 / \mathrm{embr} .201642681$

Huang J, Wu S, Barrera J, Matthews K, Pan D. 2005. The Hippo signaling pathway coordinately regulates cell proliferation and apoptosis by inactivating Yorkie, the Drosophila homolog of YAP. Cell 122: 421-434. doi:10.1016/j.cell.2005.06.007

Jia J, Zhang W, Wang B, Trinko R, Jiang J. 2003. The Drosophila Ste20 family kinase dMST functions as a tumor suppressor by restricting cell proliferation and promoting apoptosis. Genes Dev 17: 2514-2519. doi:10.1101/gad.1134003

Jiang J, Struhl G. 1995. Protein kinase A and hedgehog signaling in Drosophila limb development. Cell 80: 563-572. doi:10 .1016/0092-8674(95)90510-3

Jiang YY, Lin DC, Mayakonda A, Hazawa M, Ding LW, Chien WW, Xu L, Chen Y, Xiao JF, Senapedis W, et al. 2017. Targeting super-enhancer-associated oncogenes in oesophageal squamous cell carcinoma. Gut 66: 1358-1368. doi:10.1136/ gutjnl-2016-311818

Jiang L, Huang R, Wu Y, Diao P, Zhang W, Li J, Li Z, Wang Y, Cheng J, Yang J. 2019. Overexpression of CDK7 is associated with unfavourable prognosis in oral squamous cell carcinoma. Pathology 51: 74-80. doi:10.1016/j.pathol.2018.10.004

Kim MH, Kim J. 2017. Role of YAP/TAZ transcriptional regulators in resistance to anti-cancer therapies. Cell Mol Life Sci 74: 1457-1474. doi:10.1007/s00018-016-2412-x

Kim W, Khan SK, Gvozdenovic-Jeremic J, Kim Y, Dahlman J, Kim H, Park O, Ishitani T, Jho EH, Gao B, et al. 2017. Hippo signaling interactions with $\mathrm{Wnt} / \beta$-catenin and Notch signaling repress liver tumorigenesis. J Clin Invest 127: 137-152. doi:10 $.1172 /$ JCI88486

Kim W, Khan SK, Liu Y, Xu R, Park O, He Y, Cha B, Gao B, Yang Y. 2018. Hepatic Hippo signaling inhibits protumoural microenvironment to suppress hepatocellular carcinoma. Gut 67: 1692-1703. doi:10.1136/gutjnl-2017-314061

Koo JH, Guan KL. 2018. Interplay between YAP/TAZ and metabolism. Cell Metab 28: 196-206. doi:10.1016/j.cmet.2018.07 .010

Kwiatkowski N, Zhang T, Rahl PB, Abraham BJ, Reddy J, Ficarro SB, Dastur A, Amzallag A, Ramaswamy S, Tesar B, et al. 2014. Targeting transcription regulation in cancer with a covalent CDK7 inhibitor. Nature 511: 616-620. doi:10.1038/ nature 13393

Larochelle S, Chen J, Knights R, Pandur J, Morcillo P, ErdjumentBromage H, Tempst P, Suter B, Fisher RP. 2001. T-loop phosphorylation stabilizes the CDK7-cyclin H-MAT1 complex in vivo and regulates its CTD kinase activity. EMBO I 20: 3749 3759. doi:10.1093/emboj/20.14.3749

Lee J, Zhou P. 2007. DCAFs, the missing link of the CUL4-DDB1 ubiquitin ligase. Mol Cell 26: 775-780. doi:10.1016/j.molcel 2007.06.001

Li S, Cho YS, Yue T, Ip YT, Jiang J. 2015. Overlapping functions of the MAP4K family kinases Hppy and Msn in Hippo signaling. Cell Discov 1: 15038. doi:10.1038/celldisc.2015.38

Li B, Ni Chonghaile T, Fan Y, Madden SF, Klinger R, O'Connor AE, Walsh L, O'Hurley G, Mallya Udupi G, Joseph J, et al. 2017. Therapeutic rationale to target highly expressed CDK7 conferring poor outcomes in triple-negative breast cancer. 
Cancer Res 77: 3834-3845. doi:10.1158/0008-5472.CAN-162546

Li S, Cho YS, Wang B, Li S, Jiang J. 2018. Regulation of smoothened ubiquitylation and cell surface expression through a Cul4-DDB1-Gß E3 ubiquitin ligase complex. J Cell Sci 131: jcs218016.

Lu L, Li Y, Kim SM, Bossuyt W, Liu P, Qiu Q, Wang Y, Halder G, Finegold MJ, Lee JS, et al. 2010. Hippo signaling is a potent in vivo growth and tumor suppressor pathway in the mammalian liver. Proc Nat1 Acad Sci 107: 1437-1442. doi:10.1073/pnas .0911427107

Meng Z, Moroishi T, Mottier-Pavie V, Plouffe SW, Hansen CG, Hong AW, Park HW, Mo JS, Lu W, Lu S, et al. 2015. MAP4K family kinases act in parallel to MST1/2 to activate LATS1/ 2 in the Hippo pathway. Nat Commun 6: 8357. doi:10.1038/ ncomms 9357

Mo JS, Yu FX, Gong R, Brown JH, Guan KL. 2012. Regulation of the Hippo-YAP pathway by protease-activated receptors (PARs). Genes Dev 26: 2138-2143. doi:10.1101/gad.197582 .112

Moon S, Kim W, Kim S, Kim Y, Song Y, Bilousov O, Kim J, Lee T, Cha B, Kim M, et al. 2017. Phosphorylation by NLK inhibits YAP-14-3-3-interactions and induces its nuclear localization. $E M B O$ Rep 18: 61-71. doi:10.15252/embr .201642683

Morishita J, Kang MJ, Fidelin K, Ryoo HD. 2013. CDK7 regulates the mitochondrial localization of a tail-anchored proapoptotic protein, Hid. Cell Rep 5: 1481-1488. doi:10.1016/j.celrep.2013 .11 .030

Moya IM, Halder G. 2019. Hippo-YAP/TAZ signalling in organ regeneration and regenerative medicine. Nat Rev Mol Cell Biol 20: 211-226. doi:10.1038/s41580-018-0086-y

Nagaraja S, Vitanza NA, Woo PJ, Taylor KR, Liu F, Zhang L, Li M, Meng W, Ponnuswami A, Sun W, et al. 2017. Transcriptional dependencies in diffuse intrinsic pontine glioma. Cancer Cell 31: 635-652.e6. doi:10.1016/j.ccell.2017.03.011

$\mathrm{Oh} \mathrm{H}$, Irvine KD. 2008. In vivo regulation of Yorkie phosphorylation and localization. Development 135: 1081-1088. doi:10 $.1242 /$ dev.015255

Pan D. 2007. Hippo signaling in organ size control. Genes Dev 21: 886-897. doi:10.1101/gad.1536007

Pantalacci S, Tapon N, Léopold P. 2003. The Salvador partner Hippo promotes apoptosis and cell-cycle exit in Drosophila. Nat Cell Biol 5: 921-927. doi:10.1038/ncb1051

Patrón LA, Nagatomo K, Eves DT, Imad M, Young K, Torvund M, Guo X, Rogers GC, Zinsmaier KE. 2019. Cul4 ubiquitin ligase cofactor DCAF12 promotes neurotransmitter release and homeostatic plasticity. J Cell Biol 218: 993-1010. doi:10.1083/ jcb.201805099

Ren F, Zhang L, Jiang J. 2010. Hippo signaling regulates Yorkie nuclear localization and activity through 14-3-3 dependent and independent mechanisms. Dev Biol 337: 303-312. doi:10.1016/j.ydbio.2009.10.046

Ryoo HD, Bergmann A, Gonen H, Ciechanover A, Steller H. 2002. Regulation of Drosophila IAP1 degradation and apoptosis by reaper and ubcD1. Nat Cell Biol 4: 432-438. doi:10.1038/ ncb795

Sanchez-Vega F, Mina M, Armenia J, Chatila WK, Luna A, La KC, Dimitriadoy S, Liu DL, Kantheti HS, Saghafinia S, et al. 2018. Oncogenic signaling pathways in the cancer genome atlas. Cell 173: 321-337.e10. doi:10.1016/j.cell.2018 .03 .035

Shi Q, Li S, Li S, Jiang A, Chen Y, Jiang J. 2014. Hedgehog-induced phosphorylation by CK1 sustains the activity of Ci/Gli activa- tor. Proc Natl Acad Sci 111: E5651-E5660. doi:10.1073/pnas .1416652111

Song H, Mak KK, Topol L, Yun K, Hu J, Garrett L, Chen Y, Park O, Chang J, Simpson RM, et al. 2010. Mammalian Mst1 and Mst2 kinases play essential roles in organ size control and tumor suppression. Proc Natl Acad Sci 107: 1431-1436. doi:10 $.1073 /$ pnas.0911409107

Totaro A, Panciera T, Piccolo S. 2018. YAP/TAZ upstream signals and downstream responses. Nat Cell Biol 20: 888-899. doi:10 .1038/s41556-018-0142-z

Tsang FH, Law CT, Tang TC, Cheng CL, Chin DW, Tam WV, Wei L, Wong CC, Ng IO, Wong CM. 2019. Aberrant super-enhancer landscape in human hepatocellular carcinoma. Hepatology 69: $2502-2517$.

Udan RS, Kango-Singh M, Nolo R, Tao C, Halder G. 2003. Hippo promotes proliferation arrest and apoptosis in the Salvador/ Warts pathway. Nat Cell Biol 5: 914-920. doi:10.1038/ ncb1050

Wang G, Wang B, Jiang J. 1999. Protein kinase A antagonizes Hedgehog signaling by regulating both the activator and repressor forms of Cubitus interruptus. Genes Dev 13: 28282837. doi:10.1101/gad.13.21.2828

Wang Y, Zhang T, Kwiatkowski N, Abraham BJ, Lee TI, Xie S, Yuzugullu H, Von T, Li H, Lin Z, et al. 2015. CDK7-dependent transcriptional addiction in triple-negative breast cancer. Cell 163: 174-186. doi:10.1016/j.cell.2015.08.063

Wu S, Huang J, Dong J, Pan D. 2003. hippo encodes a Ste-20 family protein kinase that restricts cell proliferation and promotes apoptosis in conjunction with salvador and warts. Cell 114: 445-456. doi:10.1016/S0092-8674|03)00549-X

Wu S, Liu Y, Zheng Y, Dong J, Pan D. 2008. The TEAD/TEF family protein Scalloped mediates transcriptional output of the Hippo growth-regulatory pathway. Dev Cell 14: 388-398. doi:10.1016/j.devcel.2008.01.007

Xu MZ, Yao TJ, Lee NP, Ng IO, Chan YT, Zender L, Lowe SW, Poon RT, Luk JM. 2009. Yes-associated protein is an independent prognostic marker in hepatocellular carcinoma. Cancer 115: 4576-4585. doi:10.1002/cncr.24495

Yu FX, Zhao B, Guan KL. 2015. Hippo pathway in organ size control, tissue homeostasis, and cancer. Cell 163: 811-828. doi:10 $.1016 /$ j.cell.2015.10.044

Yue T, Tian A, Jiang J. 2012. The cell adhesion molecule echinoid functions as a tumor suppressor and upstream regulator of the Hippo signaling pathway. Dev Cell 22: 255-267. doi:10.1016/j .devcel.2011.12.011

Zanconato F, Forcato M, Battilana G, Azzolin L, Quaranta E, Bodega B, Rosato A, Bicciato S, Cordenonsi M, Piccolo S. 2015. Genome-wide association between YAP/TAZ/TEAD and AP-1 at enhancers drives oncogenic growth. Nat Cell Biol 17: 1218-1227. doi:10.1038/ncb3216

Zanconato F, Battilana G, Cordenonsi M, Piccolo S. 2016a. YAP/ TAZ as therapeutic targets in cancer. Curr Opin Pharmacol 29: 26-33. doi:10.1016/j.coph.2016.05.002

Zanconato F, Cordenonsi M, Piccolo S. 2016b. YAP/TAZ at the roots of cancer. Cancer Cell 29: 783-803. doi:10.1016/j.ccell .2016.05.005

Zanconato F, Battilana G, Forcato M, Filippi L, Azzolin L, Manfrin A, Quaranta E, Di Biagio D, Sigismondo G, Guzzardo V, et al. 2018. Transcriptional addiction in cancer cells is mediated by YAP/TAZ through BRD4. Nat Med 24: 1599-1610. doi:10.1038/s41591-018-0158-8

Zhang L, Ren F, Zhang Q, Chen Y, Wang B, Jiang J. 2008. The TEAD/TEF family of transcription factor Scalloped mediates Hippo signaling in organ size control. Dev Cell 14: 377-387. doi:10.1016/j.devcel.2008.01.006 
Zhang L, Yue T, Jiang J. 2009. Hippo signaling pathway and organ size control. Fly 3: 68-73. doi:10.4161/fly.3.1.7788

Zhang N, Bai H, David KK, Dong J, Zheng Y, Cai J, Giovannini M, Liu P, Anders RA, Pan D. 2010. The Merlin/NF2 tumor suppressor functions through the YAP oncoprotein to regulate tissue homeostasis in mammals. Dev Cell 19: 27-38. doi:10 .1016/j.devcel.2010.06.015

Zhao B, Wei X, Li W, Udan RS, Yang Q, Kim J, Xie J, Ikenoue T, Yu J, Li L, et al. 2007. Inactivation of YAP oncoprotein by the Hippo pathway is involved in cell contact inhibition and tissue growth control. Genes Dev 21: 2747-2761. doi:10.1101/gad .1602907

Zhao B, Ye X, Yu J, Li L, Li W, Li S, Yu J, Lin JD, Wang CY, Chinnaiyan AM, et al. 2008. TEAD mediates YAP-dependent gene induction and growth control. Genes Dev 22: 1962-1971. doi:10.1101/gad.1664408

Zheng Y, Pan D. 2019. The hippo signaling pathway in development and disease. Dev Cell 50: 264-282. doi:10.1016/j .devcel.2019.06.003

Zhong L, Yang S, Jia Y, Lei K. 2018. Inhibition of cyclin-dependent kinase 7 suppresses human hepatocellular carcinoma by inducing apoptosis. I Cell Biochem 119: 9742-9751. doi:10 $.1002 /$ jcb. 27292

Zhou D, Conrad C, Xia F, Park JS, Payer B, Yin Y, Lauwers GY, Thasler W, Lee JT, Avruch J, et al. 2009. Mst1 and Mst2 maintain hepatocyte quiescence and suppress hepatocellular carcinoma development through inactivation of the Yap1 oncogene. Cancer Cell 16: 425-438. doi:10.1016/j.ccr.2009.09.026 


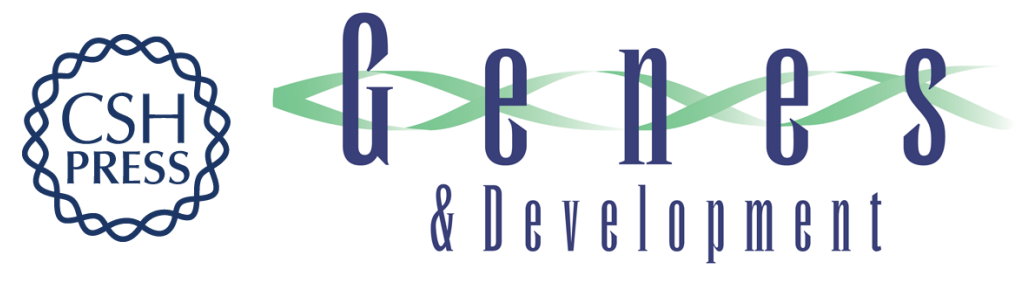

\title{
CDK7 regulates organ size and tumor growth by safeguarding the Hippo pathway effector Yki/Yap/Taz in the nucleus
}

\author{
Yong Suk Cho, Shuang Li, Xiaohui Wang, et al.
}

Genes Dev. 2020, 34: originally published online December 19, 2019

Access the most recent version at doi:10.1101/gad.333146.119

\section{Supplemental http://genesdev.cshlp.org/content/suppl/2019/12/13/gad.333146.119.DC1 \\ Material}

Related Content

References

Creative

Commons

License

Email Alerting

Service
Linking cancer transcriptional addictions by CDK7 to YAP/TAZ

Stefano Piccolo

Genes Dev. January , 2020 34: 4-6

This article cites 76 articles, 25 of which can be accessed free at:

http://genesdev.cshlp.org/content/34/1-2/53.full.html\#ref-list-1

Articles cited in:

http://genesdev.cshlp.org/content/34/1-2/53.full.html\#related-urls

This article is distributed exclusively by Cold Spring Harbor Laboratory Press for the first six months after the full-issue publication date (see

http://genesdev.cshlp.org/site/misc/terms.xhtml). After six months, it is available under a Creative Commons License (Attribution-NonCommercial 4.0 International), as described at http://creativecommons.org/licenses/by-nc/4.0/.

Receive free email alerts when new articles cite this article - sign up in the box at the top right corner of the article or click here.

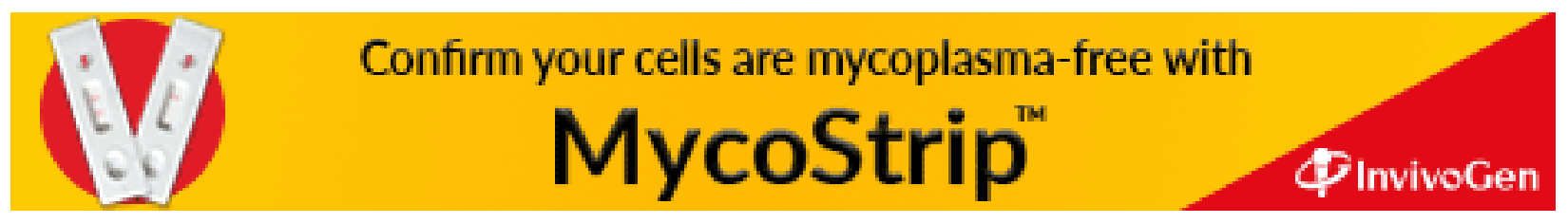

